\title{
Development of an Integrated Nonlinear Aeroservoelastic Flight Dynamic Model of the NASA Generic Transport Model
}

\author{
Nhan Nguyen * \\ NASA Ames Research Center, Moffett Field, CA 94035 \\ Eric Ting ${ }^{\dagger}$ \\ NASA Ames Research Center, Moffett Field, CA 94035
}

\begin{abstract}
This paper describes a recent development of an integrated fully coupled aeroservoelastic flight dynamic model of the NASA Generic Transport Model (GTM). The integrated model couples nonlinear flight dynamics to a nonlinear aeroelastic model of the GTM. The nonlinearity includes the coupling of the rigid-body aircraft states in the partial derivatives of the aeroelastic angle of attack. Aeroservoelastic modeling of the control surfaces which are modeled by the Variable Camber Continuous Trailing Edge Flap is also conducted. The R. T. Jones' method is implemented to approximate unsteady aerodynamics. Simulations of the GTM are conducted with simulated continuous and discrete gust loads.
\end{abstract}

\section{Introduction}

The aircraft industry has been responding to the need for energy-efficient aircraft by employing light-weight materials for aircraft structures and incorporating more energy-efficient aircraft engines. Reducing airframe operational empty weight (OEW) using advanced composite materials is one of the major considerations for improving energy efficiency. Modern light-weight materials can provide less structural rigidity while maintaining sufficient load-carrying capacity. As structural flexibility increases, aeroelastic interactions with aerodynamic forces and moments can alter aircraft aerodynamics and flight dynamics significantly, thereby potentially degrading aerodynamic efficiency, stability and control.

The general motion of a rigid-body aircraft is unconstrained in three-dimensional space as the aircraft possesses all six degrees of freedom in translation and rotation. This motion is highly influenced by all the aerodynamic forces and moments as well as the propulsive and gravity forces. The motion can exhibit stability which enables the aircraft to return to its equilibrium or trim state, or instability if the motion diverges when it is subjected to a disturbance. The general equations of motion are nonlinear, even though the aerodynamic characteristics of the aircraft are modeled as linear. It is important to recognize that a flight dynamic model is only a mathematical representation of approximate dynamics of an aircraft in flight. Many factors can cause a flight dynamic model to deviate from an observed model of an aircraft. Some of these factors are:

- Nonlinear aerodynamics - Many assumptions are usually built in the derivation of aerodynamic force and moment parameters such as coefficients and derivatives. A linear approximation is usually employed in most aerodynamic models of an aircraft. In reality, aerodynamic coefficients are not always linear and can exhibit nonlinearity at high angle of attack and sideslip angle. The linear aerodynamic approximation can provide a reasonable prediction of aircraft flight dynamics. However, in certain flight regimes, such an approximation may no longer be valid.

- Aeroelasticity - Aircraft is an elastic body which experiences stresses and strains under applied aerodynamic, propulsive, and gravity forces and moments. Elastic deformation of an aircraft results in changes in aerodynamic characteristics. Therefore, aeroelasticity is a significant effect that contributes to aircraft flight dynamics.

*NASA Ames Research Center, Research Scientist, AIAA Associate Fellow, nhan.t.nguyen@ nasa.gov

${ }^{\dagger}$ NASA Ames Research Center, Research Engineer, eric.b.ting@nasa.gov 
A rigid-body flight dynamic model is usually an initial aircraft model that an analyst develops to provide a preliminary understanding of aircraft dynamics. When a structural design is carried out, the effect of aeroelasticity must be incorporated into the rigid-body flight dynamic model in order to properly predict applied loads.

- Atmospheric disturbances - Atmospheric turbulence, wind gust, and localized weather phenomena can significantly affect aircraft dynamics. The angle of attack depends not only on the aircraft velocity vector but also the wind velocity vector. Changes in the wind velocity vector can also affect aircraft acceleration and applied loading which can cause aeroelastic responses.

The notion of a rigid-body aircraft is idealized. When aircraft structures are designed to maintain their intended aerodynamic shapes in-flight without significant deformation under aerodynamic loading, aircraft is thought of as being a rigid body and its flight characteristics are described by a six degrees of freedom flight dynamic model. As aircraft structures become increasingly more flexible, the influence of aeroelasticity becomes more pronounced. Flight control under aeroservoelastic interactions can be challenging. The mishap of the NASA Helios aircraft illustrates the complex aeroservoelasticity of flexible flight vehicles. Flight dynamics of flexible flight vehicles are intimately coupled with structural dynamics of the aircraft.

This paper describes the development of an aeroservoelastic (ASE) flight dynamic model of the NASA Generic Transport Model (GTM). ${ }^{1}$ The GTM represents a notional single-aisle, mid-size, 200-passenger transport aircraft generically approximating a Boeing 757, as shown in Fig. 1. The GTM had been extensively tested in the 14-foot-by22-foot wind tunnel at NASA Langley Research Center. Thus, wind tunnel test data are available that can be used to validate computational models. The GTM model also has been used extensive in flight control research.

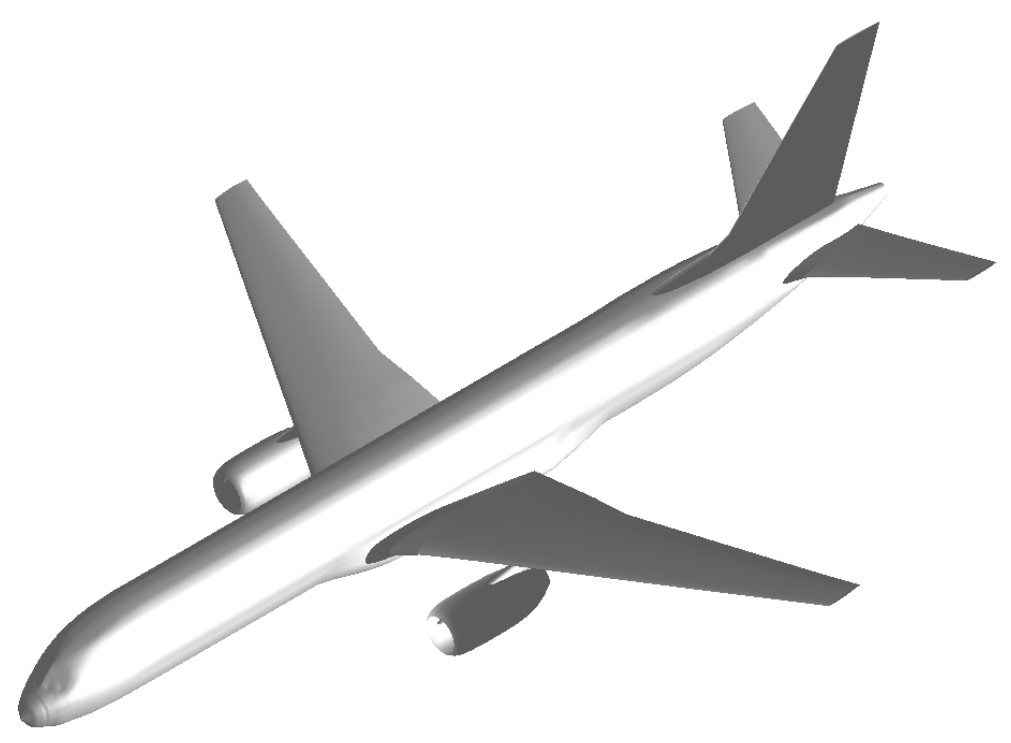

Figure 1. NASA Generic Transport Model

The aircraft has a mid-cruise weight of 210,000 lbs for a typical operating load (gear up, flap up) that includes cargo, fuel, and passengers. Fuel weighs about 50,000 lbs for a range of about 3,000 nautical miles. At the design cruise condition of Mach 0.797 at 36,000 ft, the design lift coefficient is 0.51 .

Under the Advanced Air Vehicles Program of the NASA Aeronautics Research Mission Directorate, the Advanced Air Transport Technology (AATT) Project is conducting multidisciplinary research to investigate advanced concepts and technologies for future aircraft systems. A NASA study entitled "Elastically Shaped Future Air Vehicle Concept" was conducted in $2010^{2,3}$ to examine new concepts that can enable active control of wing aeroelasticity to achieve drag reduction. This study showed that highly flexible wing aerodynamic surfaces can be elastically shaped in-flight by active control of wing twist and bending deflection in order to optimize the local angles of attack of wing sections to improve aerodynamic efficiency through drag reduction during cruise and enhance lift performance during take-off 
and landing. One concept resulting from this study is the Variable Camber Continuous Trailing Edge Flap (VCCTEF) developed initially by NASA. ${ }^{2}$ Initial study results indicate that the VCCTEF system may offer a potential pay-off in drag reduction that could provide fuel savings.

NASA and Boeing have jointly developed the VCCTEF further under a research program from 2012 to 2014. $5,6,16$ This research program was built upon the initial development of the VCCTEF system for the NASA GTM in 2010. The resulting VCCTEF system developed under this program employs light-weight Shape Memory Alloy (SMA) technology for actuation and three separate chordwise segments shaped to provide a variable camber to the flap. This cambered flap has greater potential for drag reduction as compared to a conventional straight, plain flap. The flap is also made up of individual 2-foot spanwise sections, which enable different flap settings at each flap spanwise position. This results in the ability to actively control the wing twist shape as a function of span, resulting in a change to the wing twist to establish the best lift-to-drag ratio $L / D$ at any aircraft gross weight or mission segment. Wing twist on traditional commercial transport designs is dictated by the aeroelastic deflection of a fixed "jig twist" shape applied at manufacture. The design of this jig twist is set for one cruise configuration, usually for a $50 \%$ fuel loading or mid-point on the gross weight schedule. The VCCTEF offers different wing twist settings, hence different spanwise loadings, for each gross weight condition and also different settings for climb, cruise and descent, a major factor in obtaining best $L / D$ conditions. The second feature of VCCTEF is a continuous trailing edge flap. The individual 2-foot spanwise flap sections are connected with an elastomer transition material, so as to produce no gaps in between the spanwise sections. This continuous trailing edge flap can potentially help reduce viscous drag and airframe noise. Two wind tunnel experiments were conducted for the flexible GTM wing at the University of Washington Aeronautical Laboratory in 2013 and 2014. ${ }^{7,8}$ The experimental results confirm the aerodynamic benefits of the VCCTEF.

The VCCTEF is a multi-functional flap system envisioned to be:

- A wing shaping control device to twist the flexible wing and change the span-load distribution to reduce cruise drag throughout the flight envelope,

- A high-lift device for take-off, climb-out, let-down and final approach by using the full span cambered flap,

- A full span roll control effector in lieu of traditional ailerons using the aft section of the cambered flap, and

- An aeroservoelastic (ASE) control device to compensate for reduced flutter margins of flexible wings and provide load alleviation control.

The VCCTEF is divided into 14 sections attached to the outer wing and 3 sections attached to the inner wing, as shown in Fig. $2 .^{5}$ Each 24-inch section has three chordwise cambered flap segments that can be individually commanded. These cambered flaps are joined to the next section by a flexible and supported material (shown in blue) installed with the same shape as the camber and thus providing continuous trailing edge flaps throughout the wing span with no drag producing gaps.

A major goal of the program is to develop a light-weight flap control system that has a significant weight advantage as compared to current flap screw-jack actuators. Hydraulic, electric and Shape Memory Alloy (SMA) torque rod actuation were evaluated with the result that the SMA actuation has the best weight advantage. Moreover, the use of hinge line actuation eliminates the large and heavy externally mounted actuators, and permits all actuators to be interior to the wing and flap mold lines, thus contributing to the overall drag reduction goal.

Figure 3 shows a schematic representation of an outboard wing flap section having three cambered flap segments. ${ }^{5}$ SMA actuators drive the first and second cambered flap segments and a faster acting electric actuator drives the third cambered flap segment. SMA actuators can deliver large hinge moments, but generally move at a slow rate. The outboard wing flap uses the full-span third cambered segment as a roll command effector and as a control device for suppressing aeroelastic wing structural dynamic modes, both requiring high rates which can be met by electric actuators.

Using the camber positioning, a full-span, high-lift configuration can be activated that has no drag producing gaps and a low flap noise signature. This is shown in Fig. 4. To further augment lift, a slotted Fowler flap configuration is formed by an air passage between the wing and the inner flap that serves to improve airflow over the flap and keep the flow attached. This air passage appears only when the flaps are extended in the high lift configuration.

In the high-lift configuration, the outer wing flap uses the third cambered segment for roll control, as shown in Fig. 5. This provides rolling moment that is equivalent to aileron control. It is somewhat similar to deflecting the ailerons in a droop position to act as flaps, a common procedure used on tactical aircraft and on some transport aircraft. The highlift configuration distributes the required flap hinge moment throughout the span of the wing while using actuation 
components that are all located interior to the wing and flap. This can be achieved by the use of SMA hinge line torque rods, sized to meet the hinge moment requirements at each spanwise location on the wing.

\section{EQUAL CHORD FLAP SECTIONS:}

\section{- 24" TWIST SECTIONS WITH 8" SKIN SEPARATOR SECTIONS}

- $13 \times 24$ " WIDTH - ACTUATED TRI-FLAPS

\section{- MAIN FLAP - 3 SECTIONS 75" WIDTH - NO TWIST}

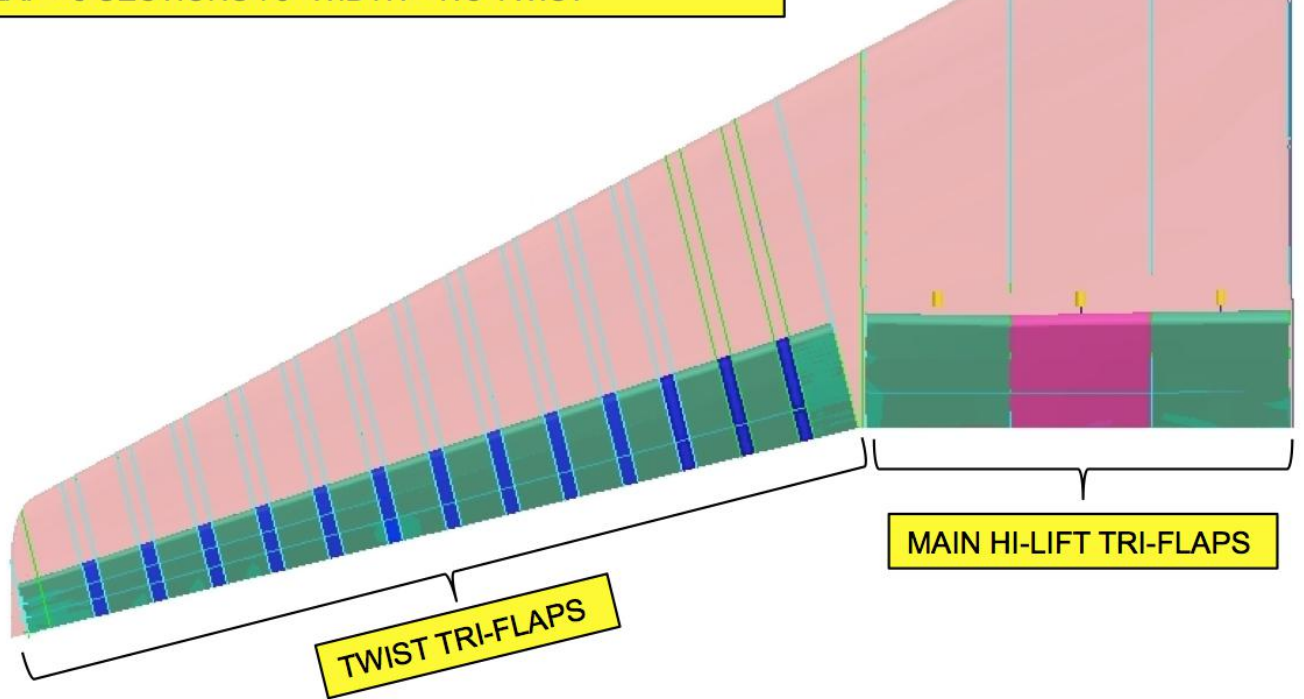

Figure 2. Wing Configured with the Variable Camber Continuous Trailing Edge Flap

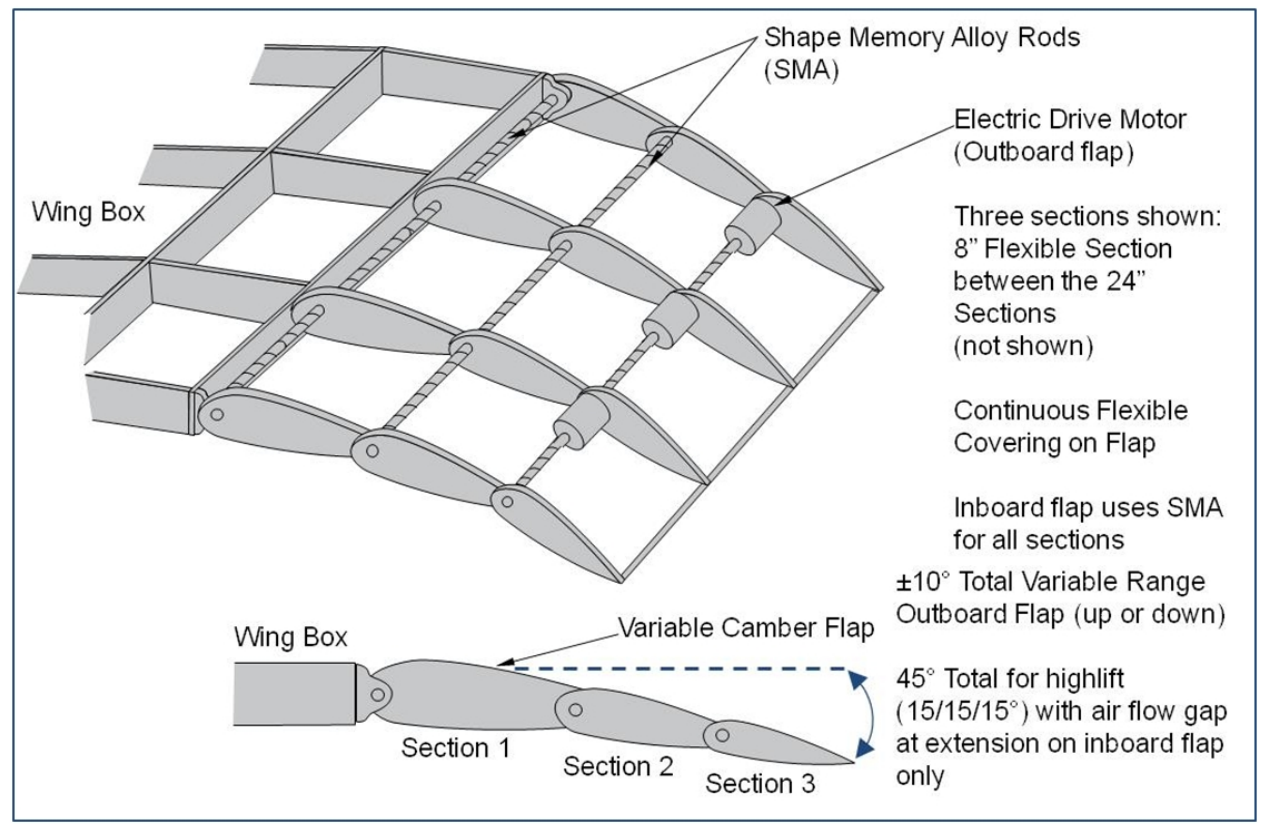

Figure 3. Variable Camber Flap Control Uses Shape Memory Alloy Torque Rod and Electric Drive Actuation 


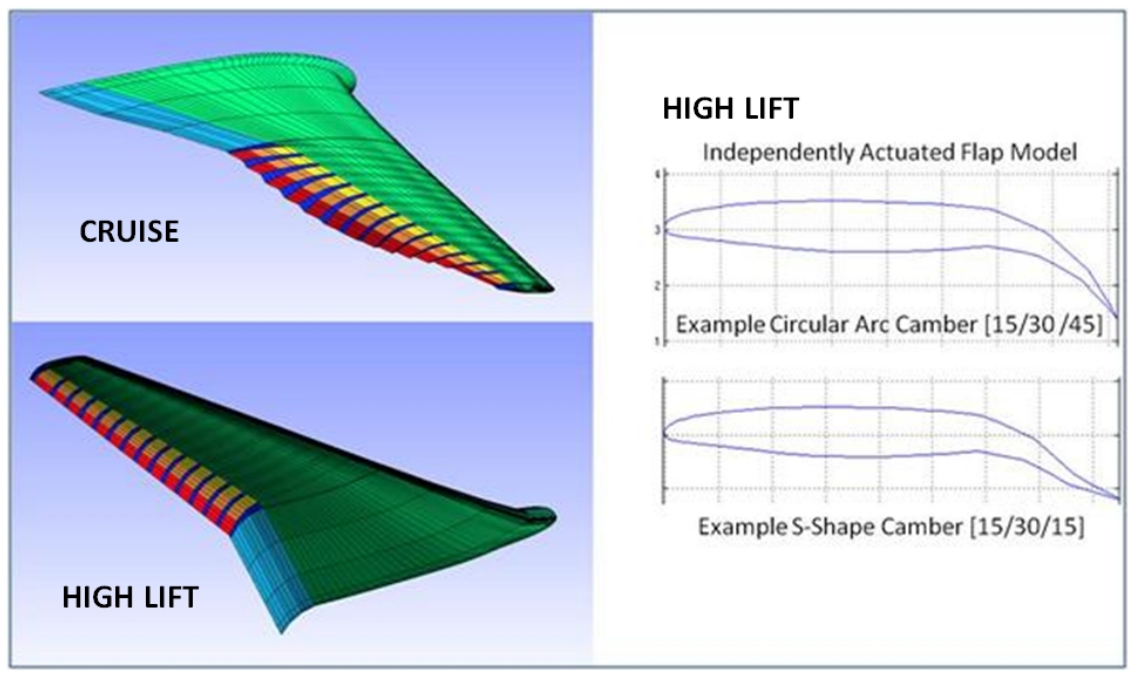

Figure 4. Cruise and High Lift VCCTEF Configurations

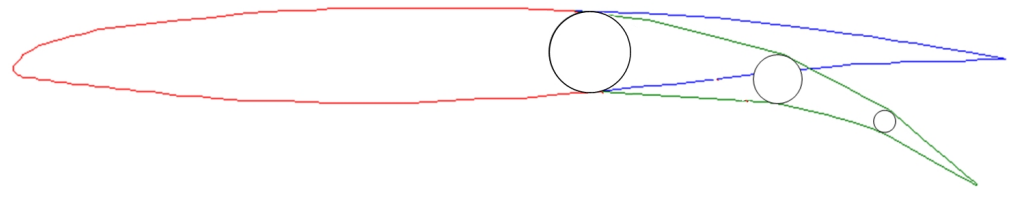

Figure 5. Three-Segment Variable Camber Flap

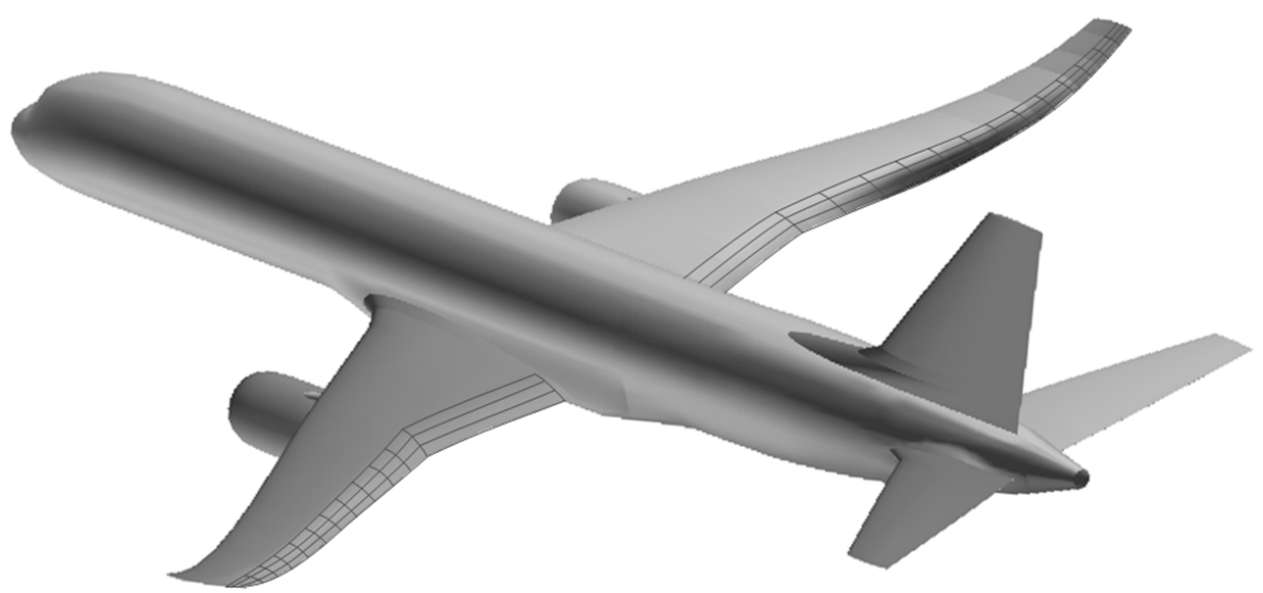

Figure 6. GTM with with Variable Camber Continuous Trailing Edge Flap 
Figure 6 illustrates the GTM equipped with the VCCTEF for wing shaping control. By actively shaping the wing aerodynamic surface using the VCCTEF, optimal aerodynamic performance could potentially be realized at any point in the flight envelope, thereby enabling a mission adaptive capability. It is a key enabling feature of the research area Performance Adaptive Aeroelastic Wing (PAAW) in the AATT project. The term "performance adaptive aeroelastic" distinguishes itself from the familiar term "mission adaptive" in that the effect of aeroelasticity on aerodynamic performance must be fully accounted for as is the case for modern transport design. The VCCTEF relies on two mechanisms to improve aerodynamic performance: 1) wing twist optimization for flexible wing design, and 2) variable camber and continuous trailing edge for improved aerodynamics. This technology could enable modern high-aspect ratio flexible wing aircraft with significant flexibility to adaptively change wing shapes in-flight to achieve cruise drag optimization, while at the same time satisfying operational constraints such as structural load limitations, flutter margins, gust and maneuver load responses, and others by active aeroservoelastic controls.

To assess the effectiveness of wing shaping control for modern transport aircraft, the GTM wing is modeled with a high degree of flexibility, similar to estimated flexibility distributions on state-of-the-art passenger aircraft wings. The wing bending stiffness is tailored to achieve a $10 \%$ wing tip deflection at 1-g flight conditions, which results in a bending stiffness about half that of older-generation transport wings, while the torsional stiffness is about the same. This $10 \%$ wing tip deflection is about the same as that of a modern composite high-aspect-ratio wing design in modern transport aircraft such as the Boeing 787.

\section{Flight Dynamics of Rigid Aircraft}

The development of a flight dynamic model of the GTM requires mass and inertia properties, and stability and control (S\&C) derivatives. The mass and inertia properties of the GTM are based on a Boeing's report and are modified to account for the reduced weight of the flexible wings. The S\&C derivatives are estimated using three different conceptual aerodynamic vortex-lattice codes along with analytical calculation. The three aerodynamic codes are VORLAX, AVL, and VSPAERO. The results show reasonable agreement among the four sets of estimates. Because VORLAX has the aeroelastic capability as well as transonic and boundary layer corrections, ${ }^{17}$ VORLAX results are selected for the flight dynamic model.

A static aeroelastic trim for the flexible wing GTM is developed. The trim solution calculates the angle of attack, engine thrust, and elevator deflections for various deformed GTM configurations at different fuel weight, altitude, and airspeed. The fuel weight is modeled as an added weight to the wing weight which affects the static deflection shape of the wings at 1-g cruise conditions. A finite-element model is developed to compute the static wing deflections. Once the wing deflection shape is computed, the $\mathrm{S} \& \mathrm{C}$ derivatives are evaluated for the deformed aircraft.

The nonlinear 6-degree-of-freedom flight dynamic equations of motion in the aircraft body-fixed reference frame are given by

$$
\begin{gathered}
\dot{\mathbf{p}}=\mathbf{R} \mathbf{V} \\
\dot{\Phi}=\mathbf{T} \omega \\
\frac{d(m \mathbf{V})}{d t}+\tilde{\omega} m \mathbf{V}=\mathbf{F} \\
\frac{d(\mathbf{I} \omega)}{d t}+\tilde{\omega} \mathbf{I} \omega=\mathbf{M}
\end{gathered}
$$

where $\mathbf{p}=\left[\begin{array}{lll}x & y & h\end{array}\right]^{\top}$ is the position vector, $\mathbf{V}=\left[\begin{array}{lll}u & v & w\end{array}\right]^{\top}$ is the velocity vector, $\Phi=\left[\begin{array}{lll}\phi & \theta & \psi\end{array}\right]^{\top}$ is the Euler angle vector, and $\omega=\left[\begin{array}{ccc}p & q & r\end{array}\right]^{\top}$ is the angular rate vector.

The angular rate matrix $\tilde{\omega}$ is given by

$$
\tilde{\omega}=\left[\begin{array}{ccc}
0 & -r & q \\
r & 0 & -p \\
-q & p & 0
\end{array}\right]
$$


The inertia matrix of the aircraft about the aircraft roll axis $X$, pitch axis $Y$, and yaw axis $Z$ is given by

$$
\mathbf{I}=\left[\begin{array}{ccc}
\bar{I}_{X X} & -\bar{I}_{X Y} & -\bar{I}_{X Z} \\
-\bar{I}_{X Y} & \bar{I}_{Y Y} & -\bar{I}_{Y Z} \\
-\bar{I}_{X Z} & -\bar{I}_{Y Z} & \bar{I}_{Z Z}
\end{array}\right]
$$

$\mathbf{R}$ and $\mathbf{T}$ are the rotation matrices given by

$$
\begin{gathered}
\mathbf{R}=\left[\begin{array}{ccc}
\cos \theta \cos \psi & -\cos \phi \sin \psi+\sin \phi \sin \theta \cos \psi & \sin \phi \sin \psi+\cos \phi \sin \theta \cos \psi \\
\cos \theta \sin \psi & \cos \phi \cos \psi+\sin \phi \sin \theta \sin \psi & -\sin \phi \cos \psi+\cos \phi \sin \theta \sin \psi \\
\sin \theta & -\sin \phi \cos \theta & -\cos \phi \cos \theta
\end{array}\right] \\
\mathbf{T}=\left[\begin{array}{ccc}
1 & \sin \phi \tan \theta & \cos \phi \tan \theta \\
0 & \cos \phi & -\sin \phi \\
0 & \sin \phi \sec \theta & \cos \phi \sec \theta
\end{array}\right]
\end{gathered}
$$

The force vector is given by

$$
\mathbf{F}=\left[\begin{array}{c}
X+T-m g \sin \theta \\
Y+m g \cos \theta \sin \phi \\
Z+m g \cos \theta \cos \phi
\end{array}\right] 1
$$

where $X=C_{X} q_{\infty} S$ is the axial force, $Y=C_{Y} q_{\infty} S$ is the side force, $Z=C_{Z} q_{\infty} S$ is the normal force, and $T$ is the engine thrust. The force coefficients $C_{X}$ and $C_{Z}$ are related to the lift and drag coefficients as

$$
\begin{gathered}
C_{X}=C_{L} \sin \alpha-C_{D} \cos \alpha \\
C_{Z}=-C_{L} \cos \alpha-C_{D} \sin \alpha
\end{gathered}
$$

The moment vector is given by

$$
\mathbf{M}=\left[\begin{array}{c}
l \\
m+T z_{e} \\
n
\end{array}\right]
$$

where $l$ is the rolling moment, $m$ is the pitching moment about the aircraft center of gravity (CG), $n$ is the yawing moment about the aircraft CG, and $z_{e}$ as the engine thrust offset from the aircraft CG.

\section{Inertial and Aeroelastic Forces and Moments of Flexible Wings}

Consider an airfoil section on the left wing as shown in Fig. 7 undergoing bending and torsional deflections. Let $(x, y, z)$ be the undeformed coordinates of point $\mathrm{Q}$ on a wing airfoil section in the reference frame $\mathrm{D}$ defined by unit vectors $\left(\mathbf{d}_{1}, \mathbf{d}_{2}, \mathbf{d}_{3}\right)$. Let $\mathbf{p}_{0}=x \mathbf{d}_{1}$ be a position vector along the elastic axis. Then, point $\mathrm{Q}$ is defined by a position vector $\mathbf{p}=\mathbf{p}_{0}+\mathbf{q}$ where $\mathbf{q}=y \mathbf{d}_{2}+z \mathbf{d}_{3}$ defines point $\mathrm{Q}$ in the $y-z$ plane from the elastic axis.
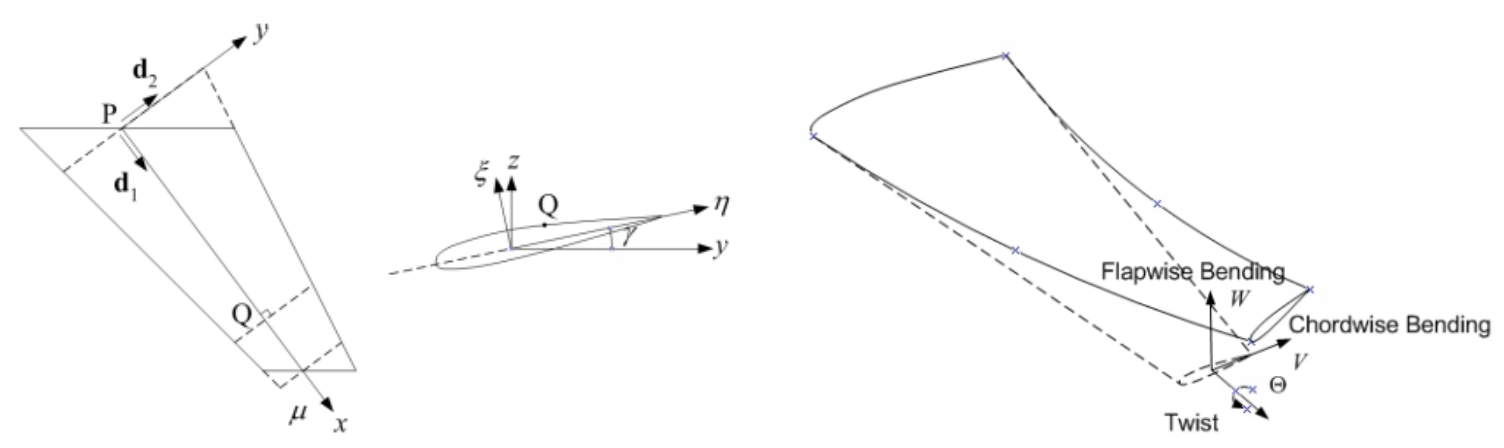

Figure 7. Left Wing Reference Frame of Wing in Combined Bending-Torsion 
Let $\Theta$ be a torsional twist angle about the $x$-axis, positive nose-down. Let $W$ and $V$ be flapwise and chordwise bending deflections of point Q, respectively. Let $U$ be the axial displacement of point $\mathrm{Q}$. Then, the displacement and rotation vectors due to the elastic deformation can be expressed as

$$
\begin{gathered}
\mathbf{r}=U \mathbf{d}_{1}+V \mathbf{d}_{2}+W \mathbf{d}_{3} \\
\phi=\Theta \mathbf{d}_{1}-W_{x} \mathbf{d}_{2}+V_{x} \mathbf{d}_{3}
\end{gathered}
$$

where the subscripts $x$ and $t$ denote the partial derivatives of $\Theta, W$, and $V$.

Let $\left(x_{1}, y_{1}, z_{1}\right)$ be the deformed coordinates of point $\mathrm{Q}$ on the airfoil in the left wing reference frame $\mathrm{D}$ and $\mathbf{p}_{1}=$ $x_{1} \mathbf{d}_{1}+y_{1} \mathbf{d}_{2}+z_{1} \mathbf{d}_{3}$ be its position vector. Then, the coordinates $\left(x_{1}, y_{1}, z_{1}\right)$ are computed as ${ }^{10}$

$$
\mathbf{p}_{1}=\mathbf{p}+\mathbf{r}+\phi \times \mathbf{q}
$$

where

$$
\left[\begin{array}{l}
x_{1} \\
y_{1} \\
z_{1}
\end{array}\right]=\left[\begin{array}{c}
x+U-y V_{x}-z W_{x} \\
y+V-z \Theta \\
z+W+y \Theta
\end{array}\right]
$$

\section{A. Inertial Forces and Moment}

Let $\mathbf{V}=u \mathbf{b}_{1}+v \mathbf{b}_{2}+w \mathbf{b}_{3}$ and $\omega=p \mathbf{b}_{1}+q \mathbf{b}_{2}+r \mathbf{b}_{3}$ be the aircraft translational and rotational velocity vectors at the aircraft center of gravity (CG) where $\left(\mathbf{b}_{1}, \mathbf{b}_{2}, \mathbf{b}_{3}\right)$ are the unit vectors in the aircraft body-fixed reference frame B. Let $\mathbf{r}_{a}=-x_{a} \mathbf{b}_{1}-y_{a} \mathbf{b}_{2}-z_{a} \mathbf{b}_{3}$ be the position vector of point $\mathrm{Q}$ in the aircraft body-fixed reference frame $\mathrm{B}$ relative to the aircraft CG such that $x_{a}$ is positive when point $\mathrm{Q}$ is aft of the aircraft CG, $y_{a}$ is positive when point $\mathrm{Q}$ is toward the left wing from the aircraft $\mathrm{CG}$, and $z_{a}$ is positive when point $\mathrm{Q}$ is above the aircraft $\mathrm{CG}$. The velocity at point $\mathrm{Q}$ due to the aircraft velocity and angular velocity in the reference frame $\mathrm{D}$ is then computed as

$$
\begin{aligned}
\mathbf{v}_{Q}=\mathbf{V}+\omega \times \mathbf{r}_{a}=\left(u \mathbf{b}_{1}\right. & \left.+v \mathbf{b}_{2}+w \mathbf{b}_{3}\right)+\left(p \mathbf{b}_{1}+q \mathbf{b}_{2}+r \mathbf{b}_{3}\right) \times\left(-x_{a} \mathbf{b}_{1}-y_{a} \mathbf{b}_{2}-z_{a} \mathbf{b}_{3}\right) \\
& =\left(u+r y_{a}-q z_{a}\right) \mathbf{b}_{1}+\left(v-r x_{a}+p z_{a}\right) \mathbf{b}_{2}+\left(w+q x_{a}-p y_{a}\right) \mathbf{b}_{3}=x_{t} \mathbf{d}_{1}+y_{t} \mathbf{d}_{2}+z_{t} \mathbf{d}_{3}
\end{aligned}
$$

where

$$
\left[\begin{array}{c}
x_{t} \\
y_{t} \\
z_{t}
\end{array}\right]=\left[\begin{array}{c}
-\left(u+r y_{a}-q z_{a}\right) \sin \Lambda \cos \Gamma-\left(v-r x_{a}+p z_{a}\right) \cos \Lambda \cos \Gamma-\left(w+q x_{a}-p y_{a}\right) \sin \Gamma \\
-\left(u+r y_{a}-q z_{a}\right) \cos \Lambda+\left(v-r x_{a}+p z_{a}\right) \sin \Lambda \\
\left(u+r y_{a}-q z_{a}\right) \sin \Lambda \sin \Gamma+\left(v-r x_{a}+p z_{a}\right) \cos \Lambda \sin \Gamma-\left(w+q x_{a}-p y_{a}\right) \cos \Gamma
\end{array}\right]
$$

The transformation between $\left(\mathbf{b}_{1}, \mathbf{b}_{2}, \mathbf{b}_{3}\right)$ and $\left(\mathbf{d}_{1}, \mathbf{d}_{2}, \mathbf{d}_{3}\right)$ is given by

$$
\begin{gathered}
{\left[\begin{array}{l}
\mathbf{b}_{1} \\
\mathbf{b}_{2} \\
\mathbf{b}_{3}
\end{array}\right]=\left[\begin{array}{ccc}
-\sin \Lambda \cos \Gamma & -\cos \Lambda & \sin \Lambda \sin \Gamma \\
-\cos \Lambda \cos \Gamma & \sin \Lambda & \cos \Lambda \sin \Gamma \\
-\sin \Gamma & 0 & -\cos \Gamma
\end{array}\right]\left[\begin{array}{l}
\mathbf{d}_{1} \\
\mathbf{d}_{2} \\
\mathbf{d}_{3}
\end{array}\right]} \\
{\left[\begin{array}{l}
\mathbf{d}_{1} \\
\mathbf{d}_{2} \\
\mathbf{d}_{3}
\end{array}\right]=\left[\begin{array}{ccc}
-\sin \Lambda \cos \Gamma & -\cos \Lambda \cos \Gamma & -\sin \Gamma \\
-\cos \Lambda & \sin \Lambda & 0 \\
\sin \Lambda \sin \Gamma & \cos \Lambda \sin \Gamma 0 & -\cos \Gamma
\end{array}\right]\left[\begin{array}{l}
\mathbf{b}_{1} \\
\mathbf{b}_{2} \\
\mathbf{b}_{3}
\end{array}\right]}
\end{gathered}
$$

The local velocity at point $\mathrm{Q}$ due to aircraft rigid-body dynamics and aeroelastic deflections in the left wing reference frame $\mathrm{D}$ is obtained as ${ }^{11,12}$

$$
\mathbf{v}=\mathbf{v}_{Q}+\frac{\partial \Delta \mathbf{p}}{\partial t}+\omega \times \Delta \mathbf{p}=v_{x} \mathbf{d}_{1}+v_{y} \mathbf{d}_{2}+v_{z} \mathbf{d}_{3}
$$


where $\Delta \mathbf{p}=\mathbf{p}_{1}-\mathbf{p}$ and

$$
\begin{gathered}
{\left[\begin{array}{c}
v_{x} \\
v_{y} \\
v_{z}
\end{array}\right]=\left[\begin{array}{c}
x_{t}+U_{t}-y V_{x t}-z W_{x t}-\omega_{z}(V-z \Theta)+\omega_{y}(W+y \Theta) \\
y_{t}+V_{t}-z \Theta_{t}+\omega_{z}\left(U-y V_{x}-z W_{x}\right)-\omega_{x}(W+y \Theta) \\
z_{t}+W_{t}+y \Theta_{t}-\omega_{y}\left(U-y V_{x}-z W_{x}\right)+\omega_{x}(V-z \Theta)
\end{array}\right]} \\
{\left[\begin{array}{c}
\omega_{x} \\
\omega_{y} \\
\omega_{z}
\end{array}\right]=\left[\begin{array}{c}
-p \sin \Lambda \cos \Gamma-q \cos \Lambda \cos \Gamma-r \sin \Gamma \\
-p \cos \Lambda+q \sin \Lambda \\
p \sin \Lambda \sin \Gamma+q \cos \Lambda \sin \Gamma-r \cos \Gamma
\end{array}\right]}
\end{gathered}
$$

The kinetic energy is formed by

$$
T=\frac{1}{2} \int \rho \mathbf{v} \cdot \mathbf{v} d A=\frac{1}{2} \int \rho\left(v_{x}^{2}+v_{y}^{2}+v_{z}^{2}\right) d A
$$

We use the method of separation of variables to express the displacements as $U(x, t)=\Phi_{u}(x) q_{u}(t), V(x, t)=$ $\Phi_{v}(x) q_{v}(t), W(x, t)=\Phi_{w}(x) q_{w}(t), \Theta(x, t)=\Phi_{\theta}(x) q_{\theta}(t)$. Then, the virtual work quantities due to the generalized coordinates $q_{u}(t), q_{v}(t), q_{w}(t)$, and $q_{\theta}(t)$ are computed in terms of the virtual displacements as ${ }^{12}$

$$
\begin{aligned}
&- f_{x}^{i} \delta U=\left[\frac{d}{d t}\left(\frac{\partial T}{\partial \dot{q}_{u}}\right)-\frac{\partial T}{\partial q_{u}}\right] \delta q_{u}=\int \rho\left[\frac{d v_{x}}{d t}-v_{y}\left(\omega_{z}+V_{x t}\right)+v_{z}\left(\omega_{y}-W_{x t}\right)\right] \delta U d A \\
&-f_{y}^{i} \delta V=\left[\frac{d}{d t}\left(\frac{\partial T}{\partial \dot{q}_{v}}\right)-\frac{\partial T}{\partial q_{v}}\right] \delta q_{v}=\int \rho\left[\frac{d v_{y}}{d t}+v_{x}\left(\omega_{z}+V_{x t}\right)-v_{z}\left(\omega_{x}+\Theta_{t}\right)\right] \delta V d A \\
&+\int \rho\left\{\frac{d\left[v_{x}(-y-V+z \Theta)+v_{y}\left(U-y V_{x}-z W_{x}\right)\right]}{d t}+v_{y}\left(y \omega_{z}+y V_{x t}\right)-v_{z}\left(y \omega_{y}-y W_{x t}\right)\right\} \delta V_{x} d A \\
&-f_{z} \delta W=\left[\frac{d}{d t}\left(\frac{\partial T}{\partial \dot{q}_{w}}\right)-\frac{\partial T}{\partial q_{w}}\right] \delta q_{w}=\int \rho\left[\frac{d v_{z}}{d t}-v_{x}\left(\omega_{y}-W_{x t}\right)+v_{y}\left(\omega_{x}+\Theta_{t}\right)\right] \delta W d A \\
&+\int \rho\left\{\frac{d\left[v_{x}(-z-W-y \Theta)+v_{z}\left(U-y V_{x}-z W_{x}\right)\right]}{d t}+v_{y}\left(z \omega_{z}+z V_{x t}\right)-v_{z}\left(z \omega_{y}-z W_{x t}\right)\right\} \delta W_{x} d A \\
&-m_{x} \delta \Theta=\left[\frac{d}{d t}\left(\frac{\partial T}{\partial \dot{q}_{\theta}}\right)-\frac{\partial T}{\partial q_{\theta}}\right] \delta q_{\theta}=\int \rho\left\{\frac{d\left[v_{y}(-z-W-y \Theta)\right]}{d t}+\frac{d\left[v_{z}(y+V-z \Theta)\right]}{d t}\right.\left.-v_{x}\left(y \omega_{y}+z \omega_{z}+z V_{x t}-y W_{x t}\right)+v_{y}\left(y \omega_{x}+y \Theta_{t}\right)+v_{z}\left(z \omega_{x}+z \Theta_{t}\right)\right\} \delta \Theta d A
\end{aligned}
$$

Let $\int y d A=A e_{c g}$ where $A=\int d A$ is the mass area and $e_{c g}$ is the offset of the CG of a wing section from the elastic axis, positive if the $\mathrm{CG}$ is aft of the elastic axis. We define $I_{x x}=\int\left(y^{2}+z^{2}\right) d A, I_{y y}=\int z^{2} d A$, and $I_{z z}=\int y^{2} d A$. Furthermore, We assume $\int z d A \approx 0$ and $I_{y z}=-\int y z d A \approx 0$. Integrating the integrals that contain $\delta V_{x}$ and $\delta W_{x}$ by parts, we obtain the linear contributions of the aeroelastic deflections to the inertial forces and moment as ${ }^{12}$

$$
\begin{array}{r}
f_{x=\rho A}^{i}=\rho-x_{t t}+y_{t} \omega_{z}-z_{t} \omega_{y}+\left(\omega_{y}^{2}+\omega_{z}^{2}\right) U+\left(\dot{\omega}_{z}-\omega_{x} \omega_{y}\right) V-\left(\dot{\omega}_{y}+\omega_{x} \omega_{z}\right) W+2 \omega_{z} V_{t}-2 \omega_{y} W_{t}+y_{t} V_{x t} \\
\left.+z_{t} W_{x t}-U_{t t}\right]+\rho A e_{c g}\left[-\left(\dot{\omega}_{y}+\omega_{x} \omega_{z}\right) \Theta-\left(\omega_{y}^{2}+\omega_{z}^{2}\right) V_{x}-2 \omega_{y} \Theta_{t}+V_{x t t}\right] \\
f_{y}^{i}=\rho A\left[-y_{t t}-x_{t} \omega_{z}+z_{t} \omega_{x}-\left(\dot{\omega}_{z}+\omega_{x} \omega_{y}\right) U+\left(\omega_{x}^{2}+\omega_{z}^{2}\right) V+\left(\dot{\omega}_{x}-\omega_{y} \omega_{z}\right) W-2 \omega_{z} U_{t}+2 \omega_{x} W_{t}+z_{t} \Theta_{t}-x_{t} V_{x t}\right. \\
\left.-V_{t t}\right]+\rho A e_{c g}\left[\left(\dot{\omega}_{x}-\omega_{y} \omega_{z}\right) \Theta+\left(\dot{\omega}_{z}+\omega_{x} \omega_{y}\right) V_{x}+2 \omega_{x} \Theta_{t}+2 \omega_{z} V_{x t}\right]+\frac{\partial}{\partial x}\left[\rho A\left(y_{t t} U-x_{t t} V+y_{t} U_{t}-x_{t} V_{t}\right)\right] \\
+\frac{\partial}{\partial x}\left\{\rho A e _ { c g } \left[-x_{t t}+y_{t} \omega_{z}-z_{t} \omega_{y}+\left(\omega_{y}^{2}+\omega_{z}^{2}\right) U+\left(\dot{\omega}_{z}-\omega_{x} \omega_{y}\right) V-\left(\dot{\omega}_{y}+\omega_{x} \omega_{z}\right) W-y_{t t} V_{x}+2 \omega_{z} V_{t}\right.\right. \\
\left.\left.-2 \omega_{y} W_{t}+z_{t} W_{x t}-U_{t t}\right]\right\}+\frac{\partial}{\partial x}\left\{\rho I_{z z}\left[-\left(\dot{\omega}_{y}+\omega_{x} \omega_{z}\right) \Theta-\left(\omega_{y}^{2}+\omega_{z}^{2}\right) V_{x}-2 \omega_{y} \Theta_{t}+V_{x t t}\right]\right\}
\end{array}
$$




$$
\begin{gathered}
f_{z}^{i}=\rho A\left[-z_{t t}+x_{t} \omega_{y}-y_{t} \omega_{x}+\left(\dot{\omega}_{y}-\omega_{x} \omega_{z}\right) U-\left(\dot{\omega}_{x}+\omega_{y} \omega_{z}\right) V+\left(\omega_{x}^{2}+\omega_{y}^{2}\right) W+2 \omega_{y} U_{t}-2 \omega_{x} V_{t}-y_{t} \Theta_{t}-x_{t} W_{x t}\right. \\
\left.-W_{t t}\right]+\rho A e_{c g}\left[\left(\omega_{x}^{2}+\omega_{y}^{2}\right) \Theta-\left(\dot{\omega}_{y}-\omega_{x} \omega_{z}\right) V_{x}-2 \omega_{y} V_{x t}-\Theta_{t t}\right]+\frac{\partial}{\partial x}\left[\rho A\left(z_{t t} U-x_{t t} W+z_{t} U_{t}-x_{t} W_{t}\right)\right] \\
+\frac{\partial}{\partial x}\left[\rho A e_{c g}\left(-x_{t t} \Theta-z_{t t} V_{x}-x_{t} \Theta_{t}-z_{t} V_{x t}\right)\right]+\frac{\partial}{\partial x}\left\{\rho I_{y y}\left[-\left(\dot{\omega}_{z}-\omega_{x} \omega_{y}\right) \Theta-\left(\omega_{y}^{2}+\omega_{z}^{2}\right) W_{x}-2 \omega_{z} \Theta_{t}+W_{x t t}\right]\right\} \\
m_{x}^{i}=\rho A\left(-z_{t t} V+y_{t t} W-z_{t} V_{t}+y_{t} W_{t}\right)+\rho A e_{c g}\left[-z_{t t}+x_{t} \omega_{y}-y_{t} \omega_{x}+\left(\dot{\omega}_{y}-\omega_{x} \omega_{z}\right) U-\left(\dot{\omega}_{x}+\omega_{y} \omega_{z}\right) V\right. \\
\left.\quad+\left(\omega_{x}^{2}+\omega_{y}^{2}\right) W+y_{t t} \Theta+2 \omega_{y} U_{t}-2 \omega_{x} V_{t}-x_{t} W_{x t}-W_{t t}\right]-\rho I_{x x} \Theta_{t t} \\
+\rho I_{y y}\left[\left(\omega_{x}^{2}+\omega_{z}^{2}\right) \Theta-\left(\dot{\omega}_{z}+\omega_{x} \omega_{y}\right) W_{x}-2 \omega_{z} W_{x t}\right]+\rho I_{z z}\left[\left(\omega_{x}^{2}+\omega_{y}^{2}\right) \Theta-\left(\dot{\omega}_{y}-\omega_{x} \omega_{z}\right) V_{x}-2 \omega_{y} V_{x t}\right]
\end{gathered}
$$

In addition to the inertial forces and pitching moment acting on a wing section, the contributions of half of the fuselage mass and inertias and the engine mass to the inertial forces and pitching moment without the rigid-body aircraft inertial force coupling are given by

$$
\begin{gathered}
\Delta f_{x}^{i}=\delta(x)\left(-\frac{1}{2} m_{f} U_{t t}\right)+\delta\left(x-x_{e}\right)\left(-m_{e} U_{t t}\right) \\
\Delta f_{y}^{i}=\delta(x)\left[-\frac{1}{2} m_{f} V_{t t}+\frac{\partial}{\partial x}\left(\frac{1}{2} I_{f, z z} V_{x t t}\right)\right]+\delta\left(x-x_{e}\right)\left[-m_{e} V_{t t}-m_{e} z_{e} \Theta_{t t}\right] \\
\Delta f_{z}^{f}=\delta(x)\left[-\frac{1}{2} m_{f} W_{t t}+\frac{1}{2} m_{f} y_{f} \Theta_{t t}+\frac{\partial}{\partial x}\left(\frac{1}{2} I_{f, y y} W_{x t t}\right)\right]+\delta\left(x-x_{e}\right)\left(-m_{e} W_{t t}+m_{e} y_{e} \Theta_{t t}\right) \\
\Delta m_{x}^{i}=\delta(x)\left(\frac{1}{2} m_{f} y_{f} W_{t t}-\frac{1}{2} I_{f, x x} \Theta_{t t}\right)+\delta\left(x-x_{e}\right)\left[m_{e} y_{e} W_{t t}-m_{e} z_{e} V_{t t}-m_{e}\left(y_{e}^{2}+z_{e}^{2}\right) \Theta_{t t}\right]
\end{gathered}
$$

where $m_{f}, I_{f, x x}, I_{f, y y}$, and $I_{f, z z}$ are the mass and inertias of the fuselage; $m_{e}$ is the mass of the engine; $y_{f}$ is the offset of the fuselage CG from the elastic axis, positive if the fuselage CG is forward of the elastic axis; $\left(x_{e}, y_{e}, z_{e}\right)$ is the coordinate of the engine $\mathrm{CG}$ in the left wing reference frame $\mathrm{D}$, positive if the engine $\mathrm{CG}$ is below and forward of the elastic axis; and $\delta(x-a)$ is the Dirac delta function which is defined as

$$
\int \delta(x-a) f(x) d x=f(a)
$$

\section{B. Aeroelastic Forces and Moment}

In order to compute the aeroelastic forces and moments, the velocity must be transformed from the left wing reference frame $\mathrm{D}$ to the airfoil local coordinate reference frame defined by $(\mu, \eta, \xi)$ as follows:

$$
\left[\begin{array}{l}
v_{\mu} \\
v_{\eta} \\
v_{\xi}
\end{array}\right]=\left[\begin{array}{ccc}
1 & 0 & 0 \\
0 & 1 & \Theta \\
0 & -\Theta & 1
\end{array}\right]\left[\begin{array}{ccc}
1 & V_{x} & 0 \\
-V_{x} & 1 & 0 \\
0 & 0 & 1
\end{array}\right]\left[\begin{array}{ccc}
1 & 0 & W_{x} \\
0 & 1 & 0 \\
-W_{x} & 0 & 1
\end{array}\right]\left[\begin{array}{c}
v_{x} \\
v_{y} \\
v_{z}
\end{array}\right] \approx\left[\begin{array}{c}
v_{x}+v_{y} V_{x}+v_{z} W_{x} \\
-v_{x} V_{x}+v_{y}+v_{z} \Theta \\
-v_{x} W_{x}-v_{y} \Theta+v_{z}
\end{array}\right]
$$

The local aeroelastic angle of attack on the airfoil section due to the velocity components $v_{\eta}$ and $v_{\xi}$ in the left wing reference frame D, as shown in Fig. 7, is computed as ${ }^{12}$

$$
\alpha_{c}=\frac{v_{\xi}}{v_{\eta}}=\frac{\bar{v}_{\xi}+\Delta v_{\xi}}{\bar{v}_{\eta}+\Delta v_{\eta}}=\frac{v_{\xi}}{\bar{v}_{\eta}}-\frac{\bar{v}_{\xi} \Delta v_{\eta}}{\bar{v}_{\eta}^{2}}
$$

where

$$
\left[\begin{array}{c}
v_{x} \\
v_{y} \\
v_{z}
\end{array}\right]=\left[\begin{array}{c}
x_{t}+U_{t}-y V_{x t}-z W_{x t}-\omega_{z}(V-z \Theta)+\omega_{y}(W+y \Theta) \\
y_{t}+V_{t}-z \Theta_{t}+\omega_{z}\left(U-y V_{x}-z W_{x}\right)-\omega_{x}(W+y \Theta) \\
z_{t}+W_{t}+y \Theta_{t}-\omega_{y}\left(U-y V_{x}-z W_{x}\right)+\omega_{x}(V-z \Theta)
\end{array}\right]
$$




$$
\begin{gathered}
\Delta v_{\xi}=-x_{t} W_{x}-y_{t} \Theta+W_{t}+y \Theta_{t}-\omega_{y}\left(U-y V_{x}-z W_{x}\right)+\omega_{x}(V-z \Theta) \\
\bar{v}_{\eta}=-u \cos \Lambda \\
\Delta v_{\eta}=y_{t}-\bar{v}_{\eta}-x_{t} V_{x}+V_{t}-z \Theta_{t}+\omega_{z}\left(U-y V_{x}-z W_{x}\right)-\omega_{x}(W+y \Theta)+z_{t} \Theta
\end{gathered}
$$

The rigid-body angle of attack is computed as

$$
\alpha_{r}(x)=-\frac{z_{t}}{u \cos \Lambda}\left(2+\frac{y_{t}}{u \cos \Lambda}\right)
$$

The elastic angle of attack is evaluated with $z=0$ as

$$
\begin{aligned}
\alpha_{e}(x, y)=\left(\frac{\omega_{y}}{u \cos \Lambda}-\frac{\omega_{z} z_{t}}{u^{2} \cos ^{2} \Lambda}\right) U-\frac{\omega_{x}}{u \cos \Lambda} V+\frac{\omega_{x} z_{t}}{u^{2} \cos ^{2} \Lambda} W+\left(\frac{y_{t}}{u \cos \Lambda}+\frac{y \omega_{x} z_{t}-z_{t}^{2}}{u^{2} \cos ^{2} \Lambda}\right) \Theta \\
\quad-\left(\frac{y \omega_{y}}{u \cos \Lambda}-\frac{y \omega_{z} z_{t}+x_{t} z_{t}}{u^{2} \cos ^{2} \Lambda}\right) V_{x}+\frac{x_{t}}{u \cos \Lambda} W_{x}-\frac{z_{t}}{u^{2} \cos ^{2} \Lambda} V_{t}-\frac{1}{u \cos \Lambda} W_{t}-\frac{y}{u \cos \Lambda} \Theta_{t}
\end{aligned}
$$

Let $y=\bar{y} \cos \Lambda$ where $\bar{y}$ is the airfoil coordinate in the streamwise direction. For circulatory lift, the elastic angle of attack is evaluated by setting $\bar{y}=e_{c}$ where $e_{c}$ is the offset of the three-quarter point from the elastic center in the streamwise direction. The circulatory lift and pitching moment coefficients are given by

$$
\begin{gathered}
c_{L_{c}}=c_{L_{\alpha}}\left[\alpha_{r}+C(k) \alpha_{e}\left(x, e_{c} \cos \Lambda\right)\right] \cos \Lambda \\
c_{m_{c}}=c_{m_{a c}}+\frac{e}{c} c_{L_{\alpha}}\left[\alpha_{r}+C(k) \alpha_{e}\left(x, e_{c} \cos \Lambda\right)\right] \cos \Lambda
\end{gathered}
$$

where $C(k)$ is the Theodorsen's function, ${ }^{13} k=\frac{\omega c}{2 u}$ is the reduced frequency, $c_{L_{\alpha}}$ is the lift curve slope, $c_{m_{a c}}$ is the pitching moment coefficient about the aerodynamic center, $c$ is the chord length in the streamwise direction, $e$ is the offset of the aerodynamic center from the elastic axis in the streamwise direction. Note that the the lift curve slope $c_{L_{\alpha}}$ also accounts for the transonic flow and viscosity in the model using a transonic small disturbance and integral boundary layer correction method. ${ }^{14}$

The non-circulatory lift and pitching moment coefficients evaluated as ${ }^{12}$

$$
\begin{gathered}
c_{L_{n c}}=\frac{\pi c \cos \Lambda}{2 V} \frac{\partial \alpha_{e}\left(x, e_{m} \cos \Lambda\right)}{\partial t} \\
c_{m_{n c}}=-2 \pi \cos \Lambda\left[\frac{c}{16} \frac{\partial \alpha_{e}}{\partial \bar{y}}+\frac{c^{2}}{128 V} \frac{\partial^{2} \alpha_{e}}{\partial \bar{y} \partial t}+\frac{e_{m}}{4 V} \frac{\partial \alpha_{e}\left(x, e_{m} \cos \Lambda\right)}{\partial t}\right]
\end{gathered}
$$

where $e_{m}$ is the offset of the mid-chord point from the elastic center in the streamwise direction.

The total lift and pitching moment coefficients are

$$
\begin{gathered}
c_{L}=c_{L_{c}}+c_{L_{n c}} \\
c_{m}=c_{m_{c}}+c_{m_{n c}}
\end{gathered}
$$

The linear aeroelastic contributions to the total drag coefficient are given by

$$
\begin{gathered}
c_{D}=c_{D_{c}}+c_{D_{n c}} \\
c_{D_{c}}=c_{D_{0}}+k c_{L_{r}}^{2}+2 k c_{L_{r}}\left(c_{L_{c}}-c_{L_{r}}\right) \\
c_{D_{n c}}=2 k c_{L_{r}} c_{L_{n c}}
\end{gathered}
$$

where $c_{L_{r}}=c_{L_{\alpha}} \alpha_{r} \cos \Lambda$, and $c_{D_{0}}$ and $k$ are drag polar parameters for a quadratic drag polar relationship. Note that the quadratic contributions of the circulatory and non-circulatory unsteady lift coefficients are assumed to be small and therefore are neglected.

The incremental aerodynamic coefficients for the aircraft are computed as

$$
\Delta C_{L}=\frac{2}{S} \int_{0}^{L}\left(c_{L}-c_{L_{r}}\right) c \cos \Lambda d x
$$




$$
\begin{gathered}
\Delta C_{D}=\frac{2}{S} \int_{0}^{L}\left(c_{D}-c_{D_{r}}\right) c \cos \Lambda d x \\
\Delta C_{Y} \approx 0 \\
\Delta C_{l}=\frac{2}{S b} \int_{0}^{L}\left[\left(c_{L_{c}}-c_{L_{r}}\right) y_{a c}+c_{L_{n c}} y_{m}\right] c \cos \Lambda d x \\
\Delta C_{m}=\frac{2}{S \bar{c}} \int_{0}^{L}\left[c_{m_{n c}} c-\left(c_{L_{c}}-c_{L_{r}}\right) x_{a c}-c_{L_{n c}} x_{m}+\left(c_{D_{c}}-c_{D_{r}}\right) z_{a c}+c_{D_{n c}} z_{m}\right] c \cos \Lambda d x \\
\Delta C_{n}=\frac{2}{S b} \int_{0}^{L}\left[-\left(c_{D_{c}}-c_{D_{r}}\right) y_{a c}-c_{D_{n c}} y_{m}\right] c \cos \Lambda d x
\end{gathered}
$$

where $c_{D_{r}}=c_{D_{0}}+k c_{L_{r}}^{2}$ is the drag coefficient due to the rigid aircraft, $\left(x_{a c}, y_{a c}, z_{a c}\right)$ is the location of the aerodynamic center from the aircraft CG in the aircraft body-fixed reference frame $\mathrm{B}$, and $\left(x_{m}, y_{m}, z_{m}\right)$ is the location of the midchord point from the aircraft CG in the aircraft body-fixed reference frame B.

The incremental aerodynamic coefficients for the aircraft can be expressed in terms of the partial derivatives as

$$
\begin{aligned}
\Delta C_{L, D, Y, l, m, n}=\frac{\partial \Delta C_{L, D, Y, l, m, n}}{\partial U} U+\frac{\partial \Delta C_{L, D, Y, l, m, n}}{\partial V} V+\frac{\partial \Delta C_{L, D, Y, l, m, n}}{\partial W} W+\frac{\partial \Delta C_{L, D, Y, l, m, n}}{\partial \Theta} \Theta+\frac{\partial \Delta C_{L, D, Y, l, m, n}}{\partial V_{x}} V_{x} \\
+ \\
+\frac{\partial \Delta C_{L, D, Y, l, m, n}}{\partial W_{x}} W_{x}+\frac{\partial \Delta C_{L, D, Y, l, m, n}}{\partial U_{t}} U_{t}+\frac{\partial \Delta C_{L, D, Y, l, m, n}}{\partial V_{t}} V_{t}+\frac{\partial \Delta C_{L, D, Y, l, m, n}}{\partial W_{t}} W_{t}+\frac{\partial \Delta C_{L, D, Y, l, m, n}}{\partial \Theta_{t}} \Theta_{t} \\
+\frac{\partial \Delta C_{L, D, Y, l, m, n}}{\partial V_{x t}} V_{x t}+\frac{\partial \Delta C_{L, D, Y, l, m, n}}{\partial W_{x t}} W_{x t}+\frac{\partial \Delta C_{L, D, Y, l, m, n}}{\partial V_{t t}} V_{t t}+\frac{\partial \Delta C_{L, D, Y, l, m, n}}{\partial W_{t t}} W_{t t}+\frac{\partial \Delta C_{L, D, Y, l, m, n}}{\partial \Theta_{t t}} \Theta_{t t}
\end{aligned}
$$

Note that $\Delta C_{L}, \Delta C_{D}$, and $\Delta C_{m}$ are non-zero for the symmetric motion while $\Delta C_{l}$ and $\Delta C_{n}$ are non-zero for the anti-symmetric motion of the flexible wings.

The total aerodynamic forces and moments due to both circulatory lift and non-circulatory lift are

$$
\begin{gathered}
f_{x}^{a}=c_{D} q_{\infty} c \sin \Lambda \cos \Lambda \\
f_{y}^{a}=c_{D} q_{\infty} c \cos ^{2} \Lambda \\
f_{z}^{a}=c_{L} q_{\infty} c \cos \Lambda \cos \Gamma \\
m_{x}^{a}=-c_{m} q_{\infty} c^{2} \cos ^{2} \Lambda \cos \Gamma \\
m_{y}^{a}=c_{m} q_{\infty} c^{2} \sin \Lambda \cos \Lambda \\
m_{z}^{a}=c_{m} q_{\infty} c^{2} \cos ^{2} \Lambda \sin \Gamma
\end{gathered}
$$

The aeroelastic equations of the wing structure are governed by

$$
\begin{gathered}
\left(E A U_{x}\right)_{x}=-f_{x}^{i}-f_{x}^{a} \\
\left(G J \Theta_{x}\right)_{x}=-m_{x}^{i}-m_{x}^{a}-\rho A e_{c g} g \\
\left(E I_{y y} W_{x x}\right)_{x x}=f_{z}^{i}+f_{z}^{i}+\frac{\partial m_{y}^{a}}{\partial x}-\rho A g \\
\left(E I_{z z} V_{x x}\right)_{x x}=f_{y}^{i}+f_{y}^{a}-\frac{\partial m_{z}^{a}}{\partial x}
\end{gathered}
$$




\section{Aerodynamic Forces and Moments due to Control Surfaces}

The motion of a control surface will influence the overall motion of a flexible aircraft. The control surface generates unsteady aerodynamic forces, pitching moment, and hinge moment as functions of the rigid-body aircraft states and the wing elastic states. The unsteady aerodynamics of a control surface can be analyzed based on the Theodorsen's theory. ${ }^{13}$ A modification to the standard Theodorsen's theory is developed for the non-circulatory hinge moment.

Consider a wing with a control surface as shown in Fig. 8. The control surface has a chord length of $c_{f}$ normal to the hinge axis. The control surface deflection $\delta$ is measured about the hinge axis. Along the streamwise direction, the control surface deflection is reduced to $\delta \cos \Lambda_{f}$. This control surface deflection and its velocity generate a downwash in the streamwise direction

$$
\Delta w=u \delta \cos \Lambda_{f}+\left(y-y_{f}\right) \dot{\delta} \cos \Lambda_{f}
$$

where $\Lambda_{f}$ is the sweep angle of the control surface hinge axis.

This downwash effectively changes the mean camber line in the streamwise direction which results in an incremental section angle of attack as

$$
\Delta \alpha=\frac{\Delta w}{u}=\delta \cos \Lambda_{f}+\frac{\left(y-y_{f}\right) \dot{\delta} \cos \Lambda_{f}}{u}
$$

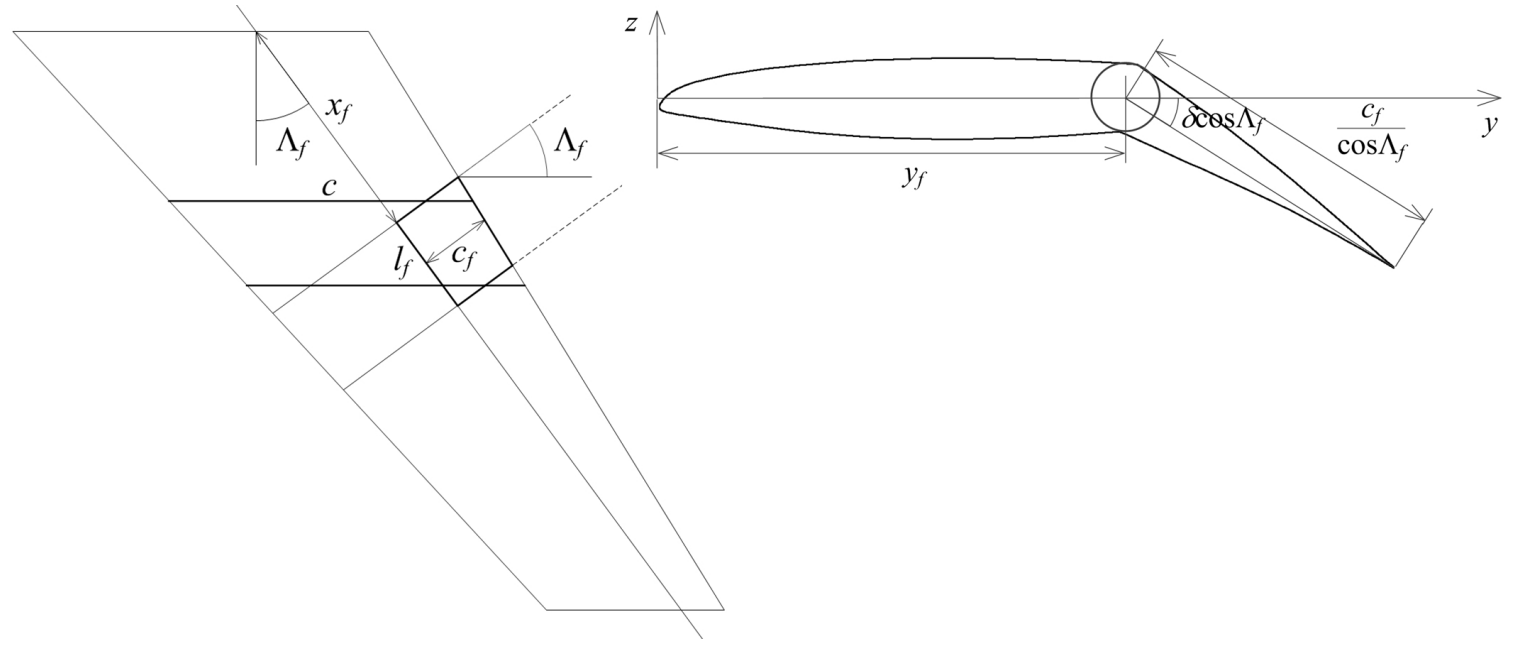

Figure 8. Wing Section with Control Surface

Let $\xi$ be the shifted normalized airfoil coordinate with the origin at the mid-chord such that $-1 \leq \xi \leq 1$ corresponding to $0 \leq y \leq c$. Then

$$
\begin{gathered}
y=\frac{c}{2}(1+\xi) \\
y_{f}=c-\frac{c_{f}}{\cos \Lambda_{f}}=\frac{c}{2}\left(1+c^{*}\right)
\end{gathered}
$$

where $\xi=c^{*}$ is at the control surface hinge location

$$
c^{*}=1-\frac{2 c_{f}}{c \cos \Lambda_{f}}
$$

Then

$$
\Delta \alpha=\delta \cos \Lambda_{f}+\frac{\left(\xi-c^{*}\right) c \dot{\delta} \cos \Lambda_{f}}{2 u}
$$




\section{A. Lift and Drag}

Based on the thin airfoil theory, we can compute the incremental section circulatory lift coefficient for incompressible flow due to the control surface deflection as

$$
\begin{aligned}
& \Delta c_{L_{c}}=\frac{C(k) c_{L_{\alpha}}}{\pi} \int_{c^{*}}^{1} \Delta \alpha \frac{(1+\xi) d \xi}{\sqrt{1-\xi^{2}}}=\frac{C(k) c_{L_{\alpha}} \delta \cos \Lambda_{f}}{\pi} \int_{c^{*}}^{1} \frac{(1+\xi) d \xi}{\sqrt{1-\xi^{2}}} \\
& +\frac{C(k) c_{L_{\alpha}} c \dot{\delta} \cos \Lambda_{f}}{2 \pi V_{\infty}} \int_{c^{*}}^{1} \frac{(1+\xi)\left(\xi-c^{*}\right) d \xi}{\sqrt{1-\xi^{2}}}
\end{aligned}
$$

Let $\xi=\cos \theta$. Then the incremental section circulatory lift coefficient is obtained as

$$
\Delta c_{L_{c}}=C(k)\left(c_{L_{\delta}} \delta+c_{L_{\dot{\delta}}} \frac{\dot{\delta} c}{2 u}\right)
$$

where $c_{L_{\delta}}$ and $c_{L_{\dot{\delta}}}$ are the section circulatory lift control derivatives evaluated as

$$
\begin{gathered}
c_{L_{\delta}}=\frac{c_{L_{\alpha}} \cos \Lambda_{f}}{\pi} T_{10} \\
c_{L_{\dot{\delta}}}=\frac{c_{L_{\alpha}} \cos \Lambda_{f}}{2 \pi} T_{11}=c_{L_{\delta}} \frac{T_{11}}{2 T_{10}}
\end{gathered}
$$

with

$$
\begin{gathered}
T_{10}=\cos ^{-1} c^{*}+\sqrt{1-c^{* 2}} \\
T_{11}=\left(1-2 c^{*}\right) \cos ^{-1} c^{*}+\left(2-c^{*}\right) \sqrt{1-c^{* 2}}
\end{gathered}
$$

Let

$$
y=\bar{y}+\frac{c}{4}+e
$$

where $\bar{y}=\frac{c}{2} \xi+e_{m}$ is the shifted airfoil coordinate with the origin at the elastic axis, $e$ is the offset of the quarter-chord and $e_{m}$ is the offset of the mid-chord from the elastic axis. Then,

$$
\Delta \alpha=\delta \cos \Lambda_{f}+\left(\frac{\bar{y}+\frac{c}{4}+e-y_{f}}{u}\right) \dot{\delta} \cos \Lambda_{f}=\Delta \bar{\alpha}+\bar{y} \frac{\partial \Delta \alpha}{\partial \bar{y}}
$$

where

$$
\begin{gathered}
\Delta \bar{\alpha}=\delta \cos \Lambda_{f}+\left(\frac{\frac{c}{4}+e-y_{f}}{u}\right) \dot{\delta} \cos \Lambda_{f}=\delta \cos \Lambda_{f}+\frac{\left(-c+4 e-2 c^{*} c\right) \dot{\delta} \cos \Lambda_{f}}{4 u} \\
\frac{\partial \Delta \alpha}{\partial \bar{y}}=\frac{\dot{\delta} \cos \Lambda_{f}}{u}
\end{gathered}
$$

The velocity potential is given by

$$
\phi=\frac{V_{\infty} c}{2}\left(\Delta \bar{\alpha}+\frac{\bar{y}+e_{m}}{2} \frac{\partial \Delta \alpha}{\partial \bar{y}}\right) \sqrt{1-\xi^{2}}
$$

The incremental section non-circulatory lift due to the control surface is computed as

$$
\Delta l_{n c}=\rho_{\infty} c \int_{c^{*}}^{1} \frac{\partial \phi}{\partial t} d \xi=\frac{\rho_{\infty} V_{\infty} c^{2}}{2} \int_{c^{*}}^{1}\left(\frac{\partial \Delta \bar{\alpha}}{\partial t}+\frac{\bar{y}+e_{m}}{2} \frac{\partial^{2} \Delta \alpha}{\partial \bar{y} \partial t}\right) \sqrt{1-\xi^{2}} d \xi
$$

The incremental section non-circulatory lift is evaluated as

$$
\begin{aligned}
\Delta l_{n c}= & \frac{\rho_{\infty} V_{\infty} c^{2} \cos \Lambda_{f}}{4}\left[\dot{\delta}+\frac{\left(-c+4 e-2 c^{*} c\right) \ddot{\delta}}{4 u}\right]\left(\cos ^{-1} c^{*}-c^{*} \sqrt{1-c^{* 2}}\right) \\
& +\frac{\rho_{\infty} V_{\infty} c^{3} \ddot{\delta} \cos \Lambda_{f}}{8 u} \frac{\left(1-c^{* 2}\right) \sqrt{1-c^{* 2}}}{3}+\frac{\rho_{\infty} V_{\infty} c^{2} e_{m} \ddot{\delta} \cos \Lambda_{f}}{4 u}\left(\cos ^{-1} c^{*}-c^{*} \sqrt{1-c^{* 2}}\right) \\
=- & \frac{\rho_{\infty} V_{\infty} c^{2} \dot{\delta} \cos \Lambda_{f}}{4}\left(-\cos ^{-1} c^{*}+c^{*} \sqrt{1-c^{* 2}}\right)-\frac{\rho_{\infty} V_{\infty} c^{3} \ddot{\delta} \cos \Lambda_{f}}{8 u}\left[c^{*} \cos ^{-1} c^{*}-\frac{\left(1+2 c^{* 2}\right) \sqrt{1-c^{* 2}}}{3}\right]
\end{aligned}
$$


The incremental section non-circulatory lift coefficient is then obtained as

$$
\Delta c_{L_{n c}}=\frac{\Delta l_{n c}}{q_{\infty} c}=\bar{c}_{L_{\dot{\delta}}} \frac{\dot{\delta} c}{2 u}+\bar{c}_{L_{\ddot{\delta}}} \frac{\ddot{\delta} c^{2}}{4 u^{2}}
$$

where

$$
\begin{aligned}
& \bar{c}_{L_{\dot{\delta}}}=-\cos \Lambda_{f} T_{4} \\
& \bar{c}_{L_{\ddot{\delta}}}=-\cos \Lambda_{f} T_{1}
\end{aligned}
$$

with

$$
\begin{gathered}
T_{1}=c^{*} \cos ^{-1} c^{*}-\frac{\left(2+c^{* 2}\right) \sqrt{1-c^{* 2}}}{3} \\
T_{4}=-\cos ^{-1} c^{*}+c^{*} \sqrt{1-c^{* 2}}
\end{gathered}
$$

The linear contributions to the incremental section drag coefficients are expressed as

$$
\begin{gathered}
\Delta c_{D_{c}}=2 k c_{L_{r}} \Delta c_{L_{c}}=C(k)\left(c_{D_{\delta}} \delta+c_{D_{\dot{\delta}}} \frac{\dot{\delta} c}{2 u}\right) \\
\Delta c_{D_{n c}}=2 k c_{L_{r}} \Delta c_{L_{n c}}=\bar{c}_{D_{\dot{\delta}}} \frac{\dot{\delta} c}{2 u}+\bar{c}_{D_{\ddot{\delta}}} \frac{\ddot{\delta} c^{2}}{4 u^{2}}
\end{gathered}
$$

\section{B. Pitching Moment about Elastic Axis}

The incremental section circulatory pitching moment coefficient about the elastic axis has two components according to the Theodorsen's theory. ${ }^{13}$ The first component is not dependent on the reduced frequency $k$ and therefore could be added to the non-circulatory pitching moment. The second component is dependent on the reduced frequency $k$ and is given by

$$
\Delta c_{m_{c}}=\frac{e}{c} \Delta c_{L}=C(k)\left(c_{m_{\delta}} \delta+c_{m_{\dot{\delta}}} \frac{\dot{\delta} c}{2 u}\right)
$$

where

$$
\begin{gathered}
c_{m_{\delta}}=\frac{c_{L_{\alpha}} e \cos \Lambda_{f}}{\pi c} T_{10} \\
c_{m_{\dot{\delta}}}=\frac{c_{L_{\alpha}} e \cos \Lambda_{f}}{2 \pi c} T_{11}=c_{m_{\delta}} \frac{T_{11}}{2 T_{10}}
\end{gathered}
$$

The incremental section non-circulatory pitching moment and the reduced frequency independent section circulatory pitching moment are given by

$$
\begin{aligned}
\Delta m_{n c} & =-\frac{q_{\infty} c^{2} \Delta c_{L}}{4}+\rho_{\infty} V_{\infty} c \int_{c^{*}}^{1} \phi d \xi-\rho_{\infty} c \int_{c^{*}}^{1} \bar{y} \frac{\partial \phi}{\partial t} d \xi=-\frac{q_{\infty} c^{2} \Delta c_{L}}{4} \\
& +\frac{\rho_{\infty} V_{\infty}^{2} c^{2}}{2} \int_{c^{*}}^{1}\left(\Delta \bar{\alpha}+\frac{\bar{y}+e_{m}}{2} \frac{\partial \Delta \alpha}{\partial \bar{y}}\right) \sqrt{1-\xi^{2}} d \xi-\frac{\rho_{\infty} V_{\infty} c^{2}}{2} \int_{c^{*}}^{1} \bar{y}\left(\frac{\partial \Delta \bar{\alpha}}{\partial t}+\frac{\bar{y}+e_{m}}{2} \frac{\partial^{2} \Delta \alpha}{\partial \bar{y} \partial t}\right) \sqrt{1-\xi^{2}} d \xi
\end{aligned}
$$

The first term is the reduced frequency independent section circulatory pitching moment. Then, the incremental section pitching moment coefficient due to the non-circulatory and reduced frequency independent section circulatory pitching moments is obtained as

$$
\Delta c_{m_{n c}}=\frac{\Delta m_{n c}}{q_{\infty} c^{2}}=\bar{c}_{m_{\delta}} \delta+\bar{c}_{m_{\dot{\delta}}} \frac{\dot{\delta} c}{2 u}+\bar{c}_{m_{\ddot{\delta}}} \frac{\ddot{\delta} c^{2}}{4 u^{2}}
$$

where

$$
\bar{c}_{m_{\delta}}=-\frac{c_{L_{\alpha}} \cos \Lambda_{f}}{4 \pi} T_{10}-\frac{\cos \Lambda_{f}}{2} T_{4}=-\frac{c_{L_{\delta}}}{4}+\frac{\bar{c}_{L_{\dot{\delta}}}}{2}
$$




$$
\begin{aligned}
& \bar{c}_{m_{\dot{\delta}}}=-\frac{c_{L_{\alpha}} \cos \Lambda_{f}}{8 \pi} T_{11}-\frac{\cos \Lambda_{f}}{2} T_{1}+\frac{\cos \Lambda_{f}}{2}\left(c^{*}+\frac{2 e_{m}}{c}\right) T_{4}+\frac{\cos \Lambda_{f}}{2} T_{8} \\
&=-\frac{c_{L_{\dot{\delta}}}}{4}+\frac{\bar{c}_{L_{\tilde{\delta}}}}{2}-\left(\frac{c^{*}}{2}+\frac{e_{m}}{c}\right) \bar{c}_{L_{\dot{\delta}}}+\frac{\cos \Lambda_{f}}{2} T_{8} \\
& \bar{c}_{m_{\ddot{\delta}}}=\frac{\cos \Lambda_{f}}{2}\left(c^{*}+\frac{2 e_{m}}{c}\right) T_{1}+\frac{\cos \Lambda_{f}}{2} T_{7}=-\left(\frac{c^{*}}{2}+\frac{e_{m}}{c}\right) \bar{c}_{L_{\ddot{\delta}}}+\frac{\cos \Lambda_{f}}{2} T_{7}
\end{aligned}
$$

with

$$
\begin{gathered}
T_{7}=-\frac{\left(1+8 c^{* 2}\right) \cos ^{-1} c^{*}}{8}+\frac{c^{*}\left(7+2 c^{* 2}\right) \sqrt{1-c^{* 2}}}{8} \\
T_{8}=c^{*} \cos ^{-1} c^{*}-\frac{\left(1+2 c^{* 2}\right) \sqrt{1-c^{* 2}}}{3}
\end{gathered}
$$

\section{Hinge Moment}

The section circulatory hinge moment coefficient about the elastic axis has two components according to the Theodorsen's theory ${ }^{13}$ The first component is not dependent on the reduced frequency $k$ and therefore could be added to the non-circulatory hinge moment. The second component is dependent on the reduced frequency $k$ and is given by Theodorsen ${ }^{13}$ as

$$
\begin{gathered}
h=-\frac{q_{\infty} c^{2} c_{L_{\alpha}}\left[\alpha\left(x, e_{c}\right)+\Delta \alpha\right]}{4 \pi} C(k) T_{12} \\
T_{12}=\left(2+c^{*}\right) \sqrt{1-c^{* 2}}-\left(1+2 c^{*}\right) \cos ^{-1} c^{*}
\end{gathered}
$$

The reduced frequency dependent section circulatory hinge moment coefficient is then obtained as

$$
c_{h_{c}}=C(k)\left[c_{h_{\alpha}} \alpha\left(x, e_{c}\right)+c_{h_{\delta}} \delta+c_{h_{\dot{\delta}}} \frac{\dot{\delta} c}{2 V_{\infty}}\right]
$$

where

$$
\begin{gathered}
c_{h_{\alpha}}=-\frac{c_{L_{\alpha}}}{4 \pi} T_{12} \\
c_{h_{\delta}}=-\frac{c_{L_{\alpha}} \cos \Lambda_{f}}{4 \pi^{2}} T_{10} T_{12}=-\frac{c_{L_{\delta}}}{4 \pi} T_{12} \\
c_{h_{\dot{\delta}}}=-\frac{c_{L_{\alpha}} \cos \Lambda_{f}}{8 \pi^{2}} T_{11} T_{12}=-\frac{c_{L_{\dot{\delta}}}}{4 \pi} T_{12}=c_{h_{\delta}} \frac{T_{11}}{2 T_{10}}
\end{gathered}
$$

The elastic angle of attack in the streamwise direction is given by

$$
\Delta \alpha(x, \bar{y})=\left(\frac{\partial \alpha_{e}}{\partial U} U+\frac{\partial \alpha_{e}}{\partial V} V+\frac{\partial \alpha_{e}}{\partial W} W+\frac{\partial \alpha_{e}}{\partial \Theta} \Theta+\frac{\partial \alpha_{e}}{\partial V_{x}} V_{x}+\frac{\partial \alpha_{e}}{\partial W_{x}} W_{x}+\frac{\partial \alpha_{e}}{\partial V_{t}} V_{t}+\frac{\partial \alpha_{e}}{\partial W_{t}} W_{t}+\frac{\partial \alpha_{e}}{\partial \Theta_{t}} \Theta_{t}\right) \cos \Lambda
$$

where $\alpha_{e}$ is the elastic angle of attack in the normal direction to the elastic axis. Note that the contributions of the rigid-body aircraft states such as aircraft angular rates $p, q, r$ are implicitly included in the elastic angle of attack $\alpha_{e}$. This allows the influences of the aircraft rigid-body motion to enter into the hinge moment expression. The elastic angle of attack can be expressed as

$$
\Delta \alpha=\Delta \bar{\alpha}+\bar{y} \frac{\partial \Delta \alpha}{\partial \bar{y}}
$$

The velocity potential is expressed as

$$
\phi=\frac{V_{\infty} c}{2}\left(\Delta \bar{\alpha}+\frac{\bar{y}+e_{m}}{2} \frac{\partial \Delta \alpha}{\partial \bar{y}}\right) \sqrt{1-\xi^{2}}
$$


The section non-circulatory hinge moment and the reduced frequency independent section circulatory hinge moment are evaluated as

$$
\begin{aligned}
h_{n c}=\frac{q_{\infty} c^{2} c_{L_{\alpha}} \Delta \alpha\left(x, e_{c}\right)}{4 \pi} T_{4}+\rho_{\infty} V_{\infty} c \int_{-1}^{1} \phi d \xi-\rho_{\infty} c \int_{-1}^{1}\left(\bar{y}-e_{m}-\frac{c c^{*}}{2}\right) \frac{\partial \phi}{\partial t} d \xi+\Delta h_{n c} \\
=\frac{q_{\infty} c^{2} c_{L_{\alpha}} \Delta \alpha\left(x, e_{c}\right)}{4 \pi} T_{4}+\frac{\rho_{\infty} V_{\infty}^{2} c^{2}}{2} \int_{c^{*}}^{1}\left(\Delta \bar{\alpha}+\frac{\bar{y}+e_{m}}{2} \frac{\partial \Delta \alpha}{\partial \bar{y}}\right) \sqrt{1-\xi^{2}} d \xi \\
\quad-\frac{\rho_{\infty} V_{\infty} c^{3}}{4} \int_{c^{*}}^{1}\left(\xi-c^{*}\right)\left(\frac{\partial \Delta \bar{\alpha}}{\partial t}+\frac{\bar{y}+e_{m}}{2} \frac{\partial^{2} \Delta \alpha}{\partial \bar{y} \partial t}\right) \sqrt{1-\xi^{2}} d \xi+\Delta h_{n c}
\end{aligned}
$$

Without derivation, the contribution to the section non-circulatory hinge moment coefficient by the control surface is given by Theodorsen as

$$
\begin{gathered}
c_{h_{n c}}=\frac{h_{n c}}{q_{\infty} c^{2}}=\bar{c}_{h_{\delta}} \delta+\bar{c}_{h_{\dot{\delta}}} \frac{\dot{\delta} c}{2 u}+\bar{c}_{h_{\ddot{\delta}}} \frac{\ddot{\delta} c^{2}}{4 u^{2}} \\
\bar{c}_{h_{\delta}}=\frac{c_{L_{\alpha}} \cos \Lambda_{f}}{4 \pi^{2}} T_{4} T_{10}-\frac{\cos \Lambda_{f}}{2 \pi} T_{5}=\frac{c_{L_{\delta}}}{4 \pi} T_{4}-\frac{\cos \Lambda_{f}}{2 \pi} T_{5}=-c_{h_{\delta}} \frac{T_{4}}{T_{12}}-\frac{\cos \Lambda_{f}}{2 \pi} T_{5} \\
\bar{c}_{h_{\dot{\delta}}}=\frac{c_{L_{\alpha}} \cos \Lambda_{f}}{8 \pi^{2}} T_{4} T_{11}=\frac{c_{L_{\dot{\delta}}}}{4 \pi} T_{4}=-c_{h_{\delta}} \frac{T_{4} T_{11}}{2 T_{10} T_{12}} \\
\bar{c}_{h_{\ddot{\delta}}}=\frac{\cos \Lambda_{f}}{2 \pi} T_{3}
\end{gathered}
$$

where

$$
\begin{gathered}
T_{3}=-\frac{\left(1+8 c^{* 2}\right)\left(\cos ^{-1} c^{*}\right)^{2}}{8}+\frac{c^{*}\left(7+2 c^{*}\right) \sqrt{1-c^{* 2}} \cos ^{-1} c^{*}}{4}-\frac{\left(4+5 c^{* 2}\right)\left(1-c^{* 2}\right)}{8} \\
T_{5}=-\left(\cos ^{-1} c^{*}\right)^{2}+2 c^{*} \sqrt{1-c^{* 2}} \cos ^{-1} c^{*}-\left(1-c^{* 2}\right)
\end{gathered}
$$

The total reduced frequency independent section hinge moment coefficient due to the section non-circulatory hinge moment and the reduced frequency independent section circulatory hinge moment is then obtained as

$$
\begin{aligned}
c_{h_{n c}}=\frac{c_{L_{\alpha}} \Delta \alpha\left(x, e_{c}\right)}{4 \pi} & T_{4}-\frac{1}{2} \Delta \bar{\alpha} T_{4}+\frac{c}{2} \frac{\partial \Delta \alpha}{\partial \bar{y}} T_{9}+\frac{c}{4 u} \frac{\partial \Delta \bar{\alpha}}{\partial t} T_{1}-\frac{c^{2}}{4 u} \frac{\partial^{2} \Delta \alpha}{\partial \bar{y} \partial t} T_{13} \\
= & \frac{T_{4} c_{L_{\alpha}} \Delta \alpha\left(x, e_{c}\right)}{4 \pi}-\frac{T_{4}}{2}\left(\Delta \bar{\alpha}-\frac{c T_{9}}{T_{4}} \frac{\partial \Delta \alpha}{\partial \bar{y}}\right)+\frac{T_{1} c}{4 u}\left(\frac{\partial \Delta \bar{\alpha}}{\partial t}-\frac{c T_{13}}{T_{1}} \frac{\partial^{2} \Delta \alpha}{\partial \bar{y} \partial t}\right) \\
= & \frac{T_{4} c_{L_{\alpha}}}{4 \pi} \Delta \alpha\left(x, e_{c}\right)-\frac{T_{4}}{2} \Delta \alpha\left(x,-\frac{c T_{9}}{T_{4}}\right)+\frac{T_{1} c}{4 u} \frac{\partial \Delta \alpha}{\partial t}\left(x,-\frac{c T_{13}}{T_{1}}\right)+\bar{c}_{h_{\delta}} \delta+\bar{c}_{h_{\dot{\delta}}} \frac{\dot{\delta} c}{2 u}+\bar{c}_{h_{\ddot{\delta}}} \frac{\ddot{\delta}^{2}}{4 u^{2}}
\end{aligned}
$$

where

$$
\begin{gathered}
p=-\frac{\left(1-c^{* 2}\right) \sqrt{1-c^{* 2}}}{3} \\
T_{9}=\frac{1}{2}\left(-p-\frac{2 e_{m}}{c} T_{4}\right) \\
T_{13}=\frac{1}{2}\left[\frac{\left(1+8 c^{* 2}\right) \cos ^{-1} c^{*}}{8}-\frac{c^{*}\left(7+2 c^{* 2}\right) \sqrt{1-c^{* 2}}}{8}-\left(c^{*}+\frac{2 e_{m}}{c}\right) T_{1}\right]=\frac{1}{2}\left[-T_{7}-\left(c^{*}+\frac{2 e_{m}}{c}\right) T_{1}\right]
\end{gathered}
$$

For theoretical $c_{L_{\alpha}}=2 \pi$, then

$$
c_{h_{n c}}=\frac{1}{2}\left(T_{9} c+T_{4} e_{c}\right) \frac{\partial \Delta \alpha}{\partial \bar{y}}+\frac{T_{1} c}{4 u} \frac{\partial \Delta \alpha}{\partial t}\left(x,-\frac{c T_{13}}{T_{1}}\right)+\bar{c}_{h_{\delta}} \delta+\bar{c}_{h_{\dot{\delta}}} \frac{\dot{\delta} c}{2 u}+\bar{c}_{h_{\ddot{\delta}}} \frac{\ddot{\delta} c^{2}}{4 u^{2}}
$$

Finally, the hinge moment about the hinge axis is evaluated as

$$
h_{f}=\left(h_{c}+h_{n c}\right) \cos \Lambda_{f}
$$


The equation of motion of the control surface is described by

$$
i_{f} \ddot{\boldsymbol{\delta}}-i_{f} \Theta_{t t}-m_{f} e_{f} W_{t t}+c_{f} \dot{\delta}+k_{f} \delta=h_{f}
$$

where $m_{f}, i_{f}, c_{f}$, and $k_{f}$ are the mass inertia, damping, and stiffness per unit length of the control surface about the hinge line, and $e_{f}$ is the offset of the control surface CG from the wing section CG, positive if the control surface CG is aft of the wing section $\mathrm{CG}$.

The contributions of the control surface to the inertial force and moment acting on a wing section are given by

$$
\begin{gathered}
\Delta f_{z}^{i}=m_{f} e_{f} \ddot{\boldsymbol{\delta}} \\
\Delta m_{x}^{i}=i_{f} \ddot{\boldsymbol{\delta}}
\end{gathered}
$$

\section{Aircraft Aerodynamic Coefficients due to Control Surfaces}

The aircraft incremental lift and drag coefficients are evaluated as

$$
\Delta C_{L, D}=C(k)\left(C_{L, D_{\delta}}+C_{L, D_{\dot{\delta}}} \frac{\dot{\delta} \bar{c}}{2 u}\right)+\bar{C}_{L, D_{\dot{\delta}}} \frac{\dot{\delta} \bar{c}}{2 u}+\bar{C}_{L, D_{\ddot{\delta}}} \frac{\ddot{\delta} \bar{c}^{2}}{4 u^{2}}
$$

where

$$
\begin{gathered}
C_{L, D_{\delta}}=\frac{1}{S} \int_{x_{f}}^{x_{f}+l_{f}} c_{L, D_{\delta}} c \cos \Lambda d x \\
C_{L, D_{\dot{\delta}}}=\frac{1}{S \bar{c}} \int_{x_{f}}^{x_{f}+l_{f}} c_{L, D_{\dot{\delta}}} c^{2} \cos \Lambda d x \\
\bar{C}_{L, D_{\dot{\delta}}}=\frac{1}{S \bar{c}} \int_{x_{f}}^{x_{f}+l_{f}} \bar{c}_{L, D_{\dot{\delta}}} c^{2} \cos \Lambda d x \\
\bar{C}_{L, D_{\ddot{\delta}}}=\frac{1}{S \bar{c}^{2}} \int_{x_{f}}^{x_{f}+l_{f}} \bar{c}_{L, D_{\ddot{\delta}}} c^{3} \cos \Lambda d x
\end{gathered}
$$

where $x_{f}$ is the coordinate of the inboard flap edge station and $l_{f}$ is the flap length.

The aircraft incremental rolling moment coefficient is evaluated as

$$
\Delta C_{l}=C(k)\left(C_{l_{\delta}}+C_{l_{\dot{\delta}}} \frac{\dot{\delta} b}{2 u}\right)+\bar{C}_{l_{\dot{\delta}}} \frac{\dot{\delta} b}{2 u}+\bar{C}_{l_{\ddot{\delta}}} \frac{\ddot{\delta} b^{2}}{4 u^{2}}
$$

where

$$
\begin{gathered}
C_{l_{\delta}}=\frac{1}{S b} \int_{x_{f}}^{x_{f}+l_{f}} c_{L_{\delta}} y_{a c} c \cos \Lambda d x \\
C_{l_{\dot{\delta}}}=\frac{1}{S b^{2}} \int_{x_{f}}^{x_{f}+l_{f}} c_{L_{\dot{\delta}}} y_{a c} c^{2} \cos \Lambda d x \\
\bar{C}_{l_{\dot{\delta}}}=\frac{1}{S b^{2}} \int_{x_{f}}^{x_{f}+l_{f}} \bar{c}_{L_{\dot{\delta}}} y_{m} c^{2} \cos \Lambda d x \\
\bar{C}_{l_{\ddot{\delta}}}=\frac{1}{S b^{3}} \int_{x_{f}}^{x_{f}+l_{f}} \bar{c}_{L_{\tilde{\delta}}} y_{m} c^{3} \cos \Lambda d x
\end{gathered}
$$

The aircraft incremental pitching moment coefficient is evaluated as

$$
\Delta C_{m}=C(k)\left(C_{m_{\delta}} \delta+C_{m_{\dot{\delta}}} \frac{\dot{\delta} \bar{c}}{2 u}\right)+\bar{C}_{m_{\delta}} \delta+\bar{C}_{m_{\dot{\delta}}} \frac{\dot{\delta} \bar{c}}{2 u}+\bar{C}_{m_{\ddot{\delta}}} \frac{\ddot{\delta} \bar{c}^{2}}{4 u^{2}}
$$

where

$$
C_{m_{\delta}}=\frac{1}{S \bar{c}} \int_{x_{f}}^{x_{f}+l_{f}}\left(-c_{L_{\delta}} x_{a c}+c_{D_{\delta}} z_{a c}\right) c \cos \Lambda d x
$$




$$
\begin{gathered}
C_{m_{\dot{\delta}}}=\frac{1}{S \bar{c}^{2}} \int_{x_{f}}^{x_{f}+l_{f}}\left(-c_{L_{\dot{\delta}}} x_{a c}+c_{D_{\dot{\delta}}} z_{a c}\right) c^{2} \cos \Lambda d x \\
\bar{C}_{m_{\delta}}=\frac{1}{S \bar{c}} \int_{x_{f}}^{x_{f}+l_{f}}\left(-\bar{c}_{L_{\delta}} x_{m}+\bar{c}_{D_{\delta}} z_{m}\right) c \cos \Lambda d x \\
\bar{C}_{m_{\dot{\delta}}}=\frac{1}{S \bar{c}^{2}} \int_{x_{f}}^{x_{f}+l_{f}}\left(-\bar{c}_{L_{\dot{\delta}}} x_{m}+\bar{c}_{D_{\dot{\delta}}} z_{m}\right) c^{2} \cos \Lambda d x \\
\bar{C}_{m_{\ddot{\delta}}}=\frac{1}{S \bar{c}^{3}} \int_{x_{f}}^{x_{f}+l_{f}}\left(-\bar{c}_{L_{\tilde{\delta}}} x_{m}+\bar{c}_{D_{\ddot{\delta}}} z_{m}\right) c^{3} \cos \Lambda d x
\end{gathered}
$$

The aircraft incremental yawing moment coefficient is evaluated as

$$
\Delta C_{n}=C(k)\left(C_{n_{\delta}}+C_{n_{\dot{\delta}}} \frac{\dot{\delta} b}{2 u}\right)+\bar{C}_{n_{\dot{\delta}}} \frac{\dot{\delta} b}{2 u}+\bar{C}_{n_{\ddot{\delta}}} \frac{\ddot{\delta} b^{2}}{4 u^{2}}
$$

where

$$
\begin{gathered}
C_{n_{\delta}}=-\frac{1}{S b} \int_{x_{f}}^{x_{f}+l_{f}} c_{D_{\delta}} y_{a c} c \cos \Lambda d x \\
C_{n_{\dot{\delta}}}=-\frac{1}{S b^{2}} \int_{x_{f}}^{x_{f}+l_{f}} c_{D_{\dot{\delta}}} y_{a c} c^{2} \cos \Lambda d x \\
\bar{C}_{n_{\dot{\delta}}}=-\frac{1}{S b^{2}} \int_{x_{f}}^{x_{f}+l_{f}} \bar{c}_{D_{\dot{\delta}}} y_{m} c^{2} \cos \Lambda d x \\
\bar{C}_{n_{\tilde{\delta}}}=-\frac{1}{S b^{3}} \int_{x_{f}}^{x_{f}+l_{f}} \bar{c}_{D_{\tilde{\delta}}} y_{m} c^{3} \cos \Lambda d x
\end{gathered}
$$

\section{Gust Models}

Atmospheric gust disturbances are an important structural design consideration for flexible wing aircraft. Gust load responses can result in structural loading issues as well as ride qualities issues relating to passenger comfort. Gust load alleviation control can reduce the gust load responses in modern transport aircraft such as Boeing 787. Two types of gust models are normally considered: discrete gust and continuous gust.

The unsteady lift due to a vertical gust velocity can be computed by the convolution integral as

$$
l_{g}=q_{\infty} c_{L_{\alpha}} c\left[\alpha_{g}(0) \psi(\tau)+\int_{0}^{\tau} \frac{d \alpha_{g}(\sigma)}{d \sigma} \psi(\tau-\sigma) d \sigma\right]
$$

where $\tau=\frac{2 V_{c} t}{c}$ is the distance in semi-chords traveled by the airfoil, $\alpha_{g}$ is the effective instantaneous angle of attack due to the vertical gust velocity, and $\psi(\tau)$ is the Küssner's function.

The instantaneous angle of attack is computed from the thin airfoil theory as

$$
\alpha_{g}(t)=\int_{0}^{\pi} w_{g}\left(t-\frac{x}{V_{\infty}}\right)(1-\cos \theta) d \theta
$$

where $x=\frac{c}{2}(1-\cos \varphi)$ is the chord distance with $x=0$ at the leading edge and $w_{g}\left(t-\frac{x}{V_{\infty}}\right)$ is a gust profile.

A more practical approach to implement a gust model is to use a differential equation instead of the convolution integral. Let $\bar{s}=\frac{s c}{2 V_{\infty}}$, then the Küssner's function can be approximated in the frequency domain using the R. T. Jones' method as ${ }^{15}$

$$
\psi(\bar{s})=1-\frac{0.500 \bar{s}}{\bar{s}+0.130}-\frac{0.500 \bar{s}}{\bar{s}+1}
$$

This can also be expressed as

$$
\psi(\bar{s})=\frac{a_{1} \bar{s}+a_{2}}{\bar{s}^{2}+a_{3} \bar{s}+a_{2}}
$$


where $a_{1}=0.565, a_{2}=0.130$, and $a_{3}=1.130$.

The asymptotic values of the Küssner's function are $\psi(\tau=0)=\psi(\bar{s} \rightarrow \infty)=0$ and $\psi(\tau \rightarrow \infty)=\psi(\bar{s}=0)=1$.

Then, the unsteady lift due to a vertical gust velocity is computed as

$$
l_{g}=q_{\infty} c_{L_{\alpha}} c \alpha_{g}(t) \psi(\bar{s})
$$

Let $y(t)=\alpha_{g}(t) \psi(\bar{s})$. Then, $y(t)$ is computed from the following differential equation

$$
\ddot{y}+a_{3}\left(\frac{2 V_{\infty}}{c}\right) \dot{y}+a_{2}\left(\frac{2 V_{\infty}}{c}\right)^{2} y=a_{1}\left(\frac{2 V_{\infty}}{c}\right) \dot{\alpha}_{g}+a_{2}\left(\frac{2 V_{\infty}}{c}\right)^{2} \alpha_{g}
$$

\section{A. Discrete Gust Model}

For a one-minus cosine gust model, the gust vertical velocity profile is given by

$$
w_{g}\left(t-\frac{x}{V_{\infty}}\right)= \begin{cases}w_{0}\left[1-\cos \frac{2 \pi}{t_{g}}\left(t-\frac{x}{V_{\infty}}\right)\right] & 0 \leq t<t_{g}, t-\frac{x}{V_{\infty}} \geq 0 \\ 0 & t \geq t_{g}, t-\frac{x}{V_{\infty}}<0\end{cases}
$$

The instantaneously angle of attack can be approximated as

$$
\alpha_{g}(\tau)=\int_{0}^{\pi} w_{g}\left(t-\frac{x}{V_{\infty}}\right)(1-\cos \theta) d \theta \approx \begin{cases}\frac{w_{0}}{V_{\infty}}\left(1-\cos \frac{2 \pi c \tau}{2 V_{\infty} t_{g}}\right) & 0 \leq \tau<\frac{2 V_{\infty} t_{g}}{c}, \tau \geq 0 \\ 0 & \tau \geq \frac{2 V_{\infty} t_{g}}{c}, \tau<0\end{cases}
$$

The incompressible Küssner's function is also given by the following R. T. Jones' approximation formula in the time domain:

$$
\psi(\tau) \approx 1-0.500 e^{-0.130 \tau}-0.500 e^{-\tau}
$$

The unsteady lift is then evaluated as

$$
l_{g}=q_{\infty} c_{L_{\alpha}} c \int_{0}^{\tau} \frac{w_{0}}{V_{\infty}}\left(1-\cos \frac{2 \pi c \sigma}{2 V_{\infty} t_{g}}\right)\left[0.065 e^{-0.130(\tau-\sigma)}+0.500 e^{-(\tau-\sigma)}\right] d \sigma
$$

The analytical expression for the unsteady lift due to the one-minus cosine gust is obtained as

$$
l_{g}=q_{\infty} c_{L_{\alpha}} c \frac{w_{0}}{V_{\infty}}\left[1-0.500 e^{-0.130 \frac{2 V_{\infty} t}{c}}-0.500 e^{-\frac{2 V_{\infty} t}{c}}-\frac{0.065\left(0.130 \cos \frac{2 \pi t}{t_{g}}+\frac{2 \pi c}{2 V_{\infty} t_{g}} \sin \frac{2 \pi t}{t_{g}}-0.130 e^{-0.130 \frac{2 V_{\infty} t}{c}}\right)}{0.130^{2}+\left(\frac{2 \pi c}{2 V_{\infty} t_{g}}\right)^{2}}\right.
$$

for $t<t_{g}$.

\section{B. Continuous Gust Model}

Two types of continuous gust models are considered: Dryden turbulence model and von Karman turbulence model.

\section{Dryden Turbulence}

The Dryden turbulence continuous gust model for the vertical velocity component is given by the power spectrum density function according to MIL-F-8785C ${ }^{16}$

$$
\Phi(\omega)=|H(\omega)|^{2}=\sigma^{2} \frac{L}{V} \frac{1+3\left(\frac{L \omega}{V}\right)^{2}}{\left[1+\left(\frac{L \omega}{V}\right)^{2}\right]^{2}}
$$


where $\sigma$ is the turbulence intensity, $L$ is the characteristic length, $V$ is the airspeed, and $\omega$ is the frequency of the spectrum.

The turbulence intensity is given by

$$
\sigma=0.1 w_{20}
$$

where $w_{20}$ is the wind speed at an altitude of $20 \mathrm{ft}$ and is equal to $15 \mathrm{knots}$ or $25.3 \mathrm{ft} / \mathrm{sec}$ for light turbulence, $30 \mathrm{knots}$ or $50.6 \mathrm{ft} / \mathrm{sec}$ for moderate turbulence, and $45 \mathrm{knots}$ or $76.0 \mathrm{ft} / \mathrm{sec}$ for severe turbulence. The characteristic length above $2000 \mathrm{ft}$ is equal to $L=1750 \mathrm{ft}$ based on MIL-F-8785C. ${ }^{16}$

The response of the vertical gust velocity to a random input is computed from the transfer function $H(\omega)$ as follows ${ }^{17}$

$$
\frac{w_{g}}{w_{i n}}=H(\omega)=\sigma \sqrt{\frac{L}{V}} \frac{1+\sqrt{3} \frac{L}{V} i \omega}{\left(1+\frac{L i \omega}{V}\right)^{2}}
$$

Let $s=i \omega$. Then,

$$
\frac{w_{g}}{w_{\text {in }}}=\sigma \sqrt{\frac{L}{V}} \frac{1+\sqrt{3} \frac{L}{V} s}{\left(1+\frac{L}{V} s\right)^{2}}
$$

The differential equation for the Dryden turbulence model is then given by

$$
\ddot{w}_{g}+2 \frac{V}{L} \dot{w}_{g}+\frac{V^{2}}{L^{2}} w_{g}=\sigma \frac{V}{L} \sqrt{\frac{V}{L}} w_{i n}+\sigma \sqrt{\frac{3 V}{L}} \dot{w}_{i n}
$$

Let

$$
w_{a}=\dot{w}_{g}-\sigma \sqrt{\frac{3 V}{L}} w_{i n}
$$

Then,

$$
\dot{w}_{a}=\ddot{w}_{g}-\sigma \sqrt{\frac{3 V}{L}} \dot{w}_{i n}=-2 \frac{V}{L} \dot{w}_{g}-\frac{V^{2}}{L^{2}} w_{g}+\sigma \frac{V}{L} \sqrt{\frac{V}{L}} w_{i n}=-2 \frac{V}{L} w_{a}-\frac{V^{2}}{L^{2}} w_{g}+\sigma \frac{V}{L} \sqrt{\frac{V}{L}}(1-2 \sqrt{3}) w_{i n}
$$

The state state form of the Dryden turbulence model is then expressed as

$$
\left[\begin{array}{c}
\dot{w}_{g} \\
\dot{w}_{a}
\end{array}\right]=\left[\begin{array}{cc}
0 & 1 \\
-\frac{V^{2}}{L^{2}} & -2 \frac{V}{L}
\end{array}\right]\left[\begin{array}{c}
w_{g} \\
w_{a}
\end{array}\right]+\sigma \sqrt{\frac{V}{L}}\left[\begin{array}{c}
\sqrt{3} \\
\frac{V}{L}(1-2 \sqrt{3})
\end{array}\right] w_{\text {in }}
$$

The input forcing function $w_{\text {in }}$ is a white noise signal which can be scaled to give a desired amplitude for the severe gust case.

\section{2. von Karman Turbulence}

The von Karman turbulence model is described by the following transfer function for the vertical gust velocity: ${ }^{17}$

$$
H(s)=\sigma \sqrt{\frac{L}{V}} \frac{1+2.7478 \frac{L}{V} s+0.3398 \frac{L^{2}}{V^{2}} s^{2}}{1+2.9958 \frac{L}{V} s+1.9754 \frac{L^{2}}{V^{2}} s^{2}+0.1539 \frac{L^{3}}{V^{3}} s^{3}}
$$

The differential equation for the Von Karman turbulence is

$$
0.1539 \frac{L^{3}}{V^{3}} \dddot{w}_{g}+1.9754 \frac{L^{2}}{V^{2}} \ddot{w}_{g}+2.9958 \frac{L}{V} \dot{w}_{g}+w_{g}=\sigma \sqrt{\frac{L}{V}} w_{i n}+2.7478 \sigma \frac{L}{V} \sqrt{\frac{L}{V}} \dot{w}_{i n}+0.3398 \sigma \frac{L^{2}}{V^{2}} \sqrt{\frac{L}{V}} \ddot{w}_{i n}
$$

or

$$
\begin{aligned}
\dddot{w}_{g}+12.8356 \frac{V}{L} \ddot{w}_{g}+19.4659 \frac{V^{2}}{L^{2}} \dot{w}_{g}+6.4977 \frac{V^{3}}{L^{3}} w_{g}=6.4977 \sigma \frac{V^{2}}{L^{2}} \sqrt{\frac{V}{L}} w_{i n}+17.85445 \sigma & \frac{V}{L} \sqrt{\frac{V}{L}} \dot{w}_{i n} \\
& +2.2079 \sigma \sqrt{\frac{V}{L}} \ddot{w}_{i n}
\end{aligned}
$$


Let

$$
\begin{gathered}
\dot{w}_{g}=-12.8356 \frac{V}{L} w_{g}+w_{a}+2.2079 \sigma \sqrt{\frac{V}{L}} w_{i n} \\
\dot{w}_{a}=-19.4659 \frac{V^{2}}{L^{2}} w_{g}+w_{b}+17.85445 \sigma \frac{V}{L} \sqrt{\frac{V}{L}} w_{i n} \\
\dot{w}_{b}=-6.4977 \frac{V^{3}}{L^{3}} w_{g}+6.4977 \sigma \frac{V^{2}}{L^{2}} \sqrt{\frac{V}{L}} w_{i n}
\end{gathered}
$$

Then,

$$
\begin{aligned}
\dddot{w}_{g}=-12.8356 \frac{V}{L} \ddot{w}_{g}+\ddot{w}_{a}+2.2079 \sigma \sqrt{\frac{V}{L}} \ddot{w}_{i n}=-12.8356 \frac{V}{L} \ddot{w}_{g}-19.4659 \frac{V^{2}}{L^{2}} \dot{w}_{g}-6.4977 \frac{V^{3}}{L^{3}} w_{g} \\
+6.4977 \sigma \frac{V^{2}}{L^{2}} \sqrt{\frac{V}{L}} w_{i n}+17.85445 \sigma \frac{V}{L} \sqrt{\frac{V}{L}} \dot{w}_{i n}+2.2079 \sigma \sqrt{\frac{V}{L}} \ddot{w}_{i n}
\end{aligned}
$$

The state space form of the von Karman turbulence is then expressed as

$$
\left[\begin{array}{c}
\dot{w}_{g} \\
\dot{w}_{a} \\
\dot{w}_{b}
\end{array}\right]=\left[\begin{array}{ccc}
-12.8356 \frac{V}{L} & 1 & 0 \\
-19.4659 \frac{V^{2}}{L^{2}} & 0 & 1 \\
-6.4977 \frac{V^{3}}{L^{3}} & 0 & 0
\end{array}\right]\left[\begin{array}{c}
w_{g} \\
w_{a} \\
w_{b}
\end{array}\right]+\sigma \sqrt{\frac{V}{L}}\left[\begin{array}{c}
2.2079 \\
17.85445 \frac{V}{L} \\
6.4977 \frac{V^{2}}{L^{2}}
\end{array}\right] w_{i n}
$$

\section{Integrated Gust Loads}

The gust model is implemented using a strip theory approach for each airfoil section of the wings and the horizontal tails. The different arrival time of the gust front at the wings and horizontal tails are are accounted in the gust model based on the wing and horizontal tail leading edge sweep angles and the distance between the quarter-chord points of the wings and the horizontal tails.

For a wing section, the incremental aerodynamic force and pitching moment coefficients are computed by

$$
\begin{gathered}
\Delta c_{L}=c_{L_{g}}=\frac{l_{g}}{q_{\infty} c} \\
\Delta c_{D}=c_{D_{g}}=k c_{L_{g}}^{2} \\
\Delta c_{m}=c_{m_{g}}=\frac{e}{c} c_{L_{g}}
\end{gathered}
$$

The integrated gust loads on the aircraft are computed as

$$
\begin{gathered}
L_{g}=q_{\infty} \int_{-L}^{L} c_{L_{g}} c \cos \Lambda d x+q_{\infty} \int_{-L_{h}}^{-L_{h}} c_{L_{g, h}} c_{h} \cos \Lambda_{h} d x \\
D_{g}=q_{\infty} \int_{-L}^{L} c_{D_{g}} c \cos \Lambda d x+q_{\infty} \int_{-L_{h}}^{-L_{h}} c_{D_{g, h}} c_{h} \cos \Lambda_{h} d x \\
l_{g}=q_{\infty} \int_{-L}^{L} c_{L_{g}} y_{a c} c \cos \Lambda d x+q_{\infty} \int_{-L_{h}}^{-L_{h}} c_{L_{g, h}} y_{a c, h} c_{h} \cos \Lambda_{h} d x \\
m_{g}=q_{\infty} \int_{-L}^{L}\left(-c_{L_{g}} x_{a c}+c_{D_{g}} z_{a c}\right) c \cos \Lambda d x+q_{\infty} \int_{-L_{h}}^{-L_{h}}\left(-c_{L_{g, h}} x_{a c, h}+c_{D_{g}} z_{a c, h}\right) c_{h} \cos \Lambda_{h} d x \\
n_{g}=-q_{\infty} \int_{-L}^{L} c_{D_{g}} y_{a c} c \cos \Lambda d x-q_{\infty} \int_{-L_{h}}^{-L_{h}} c_{D_{g, h}} y_{a c, h} c_{h} \cos \Lambda_{h} d x
\end{gathered}
$$

The incremental aerodynamic coefficients are given by

$$
\Delta C_{L}=\frac{L_{g}}{q_{\infty} S}
$$

$$
22 \text { of } 32
$$




$$
\begin{gathered}
\Delta C_{D}=\frac{D_{g}}{q_{\infty} S} \\
\Delta C_{l}=\frac{l_{g}}{q_{\infty} S b} \\
\Delta C_{m}=\frac{m_{g}}{q_{\infty} S \bar{c}} \\
\Delta C_{n}=\frac{n_{g}}{q_{\infty} S b}
\end{gathered}
$$

\section{Integrated Aeroservoelastic Flight Dynamic Model}

The aeroelastic equations can be discretized using the finite-element method (FEM). The FEM formulation results in the following global matrix equation upon assembly

$$
\begin{array}{r}
\left(\mathbf{M}_{s}+\mathbf{M}_{a n}\right) \ddot{\mathbf{q}}+\mathbf{M}_{e} \ddot{\mathbf{u}}_{e}+\left[\mathbf{C}_{s}+C(k) \mathbf{C}_{a c}+\mathbf{C}_{a n}\right] \dot{\mathbf{q}}+\left[\mathbf{K}_{s}+C(k) \mathbf{K}_{a c}\right] \mathbf{q}=\mathbf{Q}_{e} \dot{\mathbf{x}}_{r}+\mathbf{P}_{e} \mathbf{x}_{r}+\mathbf{F}_{e n} \ddot{\mathbf{u}}_{e} \\
+\left[\mathbf{E}_{e n}+C(k) \mathbf{E}_{e c}\right] \dot{\mathbf{u}}_{e}+\left[\mathbf{D}_{e n}+C(k) \mathbf{D}_{e c}\right] \mathbf{u}_{e}+\mathbf{f}_{e g}
\end{array}
$$

where $\mathbf{x}_{r}=\left[\begin{array}{llllllllllll}x & y & h & \phi & \theta & \psi & u & v & w & p & q & r\end{array}\right]^{\top}$ is the rigid-body aircraft state vector, $\mathbf{q}$ is the generalized coordinate vector, $\mathbf{u}_{e}=\left[\begin{array}{llll}\delta_{1} & \delta_{2} & \ldots & \delta_{N}\end{array}\right]^{\top}$ is a vector of the VCCTEF deflections of the trailing edge cambered segments along each of the wings, the subscript $s$ denotes a structural matrix, the subscript an denotes a reduced frequency independent non-circulatory aerodynamic matrix, the subscript $a c$ denotes a reduced frequency dependent circulatory aerodynamic matrix, the subscript en denotes a reduced frequency independent non-circulatory matrix associated with the control surface motion, and the subscript $e c$ denotes a reduced frequency dependent circulatory matrix associated with the control surface motion. Note that the aerodynamic mass matrix $\mathbf{M}_{a n}$, damping matrices $\mathbf{C}_{a c}$ and $\mathbf{C}_{a n}$, and stiffness matrix $\mathbf{K}_{a c}$ are functions of the rigid-body state vector $\mathbf{x}_{r}$ and its derivative $\dot{\mathbf{x}}_{r}$ due to the inertial coupling to the aeroelastic deflections. The aeroelastic equations also include the generalized force due to the gust $\mathbf{f}_{e g}$. Thus, the equation is nonlinear with respect to the rigid-body states even though the aeroelastic equation is linear in terms of structural deflections.

The circulatory matrices are functions of the reduced frequency parameter $k$. This form of equations is useful for flutter analyses since $k$ is usually computed from a flutter solution. For flight dynamic modeling, when a wing is excited by multiple frequencies such as gust loads, the reduced frequency dependent equations is generally inconvenient since the Theodorsen's function can only handle a single value of the reduced frequency. Therefore, the R. T. Jones method of unsteady aerodynamic approximation can be used to convert the reduced frequency dependent matrix equation into a reduced frequency independent form. The R. T. Jones method is a second-order rational fraction approximation of the Theodorsen's function according to ${ }^{18}$

$$
C(k) \approx \bar{C}(\bar{s})=\frac{0.5 \bar{s}^{2}+a_{1} \bar{s}+a_{2}}{\bar{s}^{2}+a_{3} \bar{s}+a_{2}}
$$

where $\bar{C}(s)$ is an approximate Theodorsen's function $C(k)$ which is equal to unity at $s=0$ and 0.5 as $s \rightarrow \infty, a_{1}=$ $0.2808, a_{2}=0.01365, a_{3}=0.3455$, and $\bar{s}=\frac{s \bar{c}}{2 u}$ is a dimensionless Laplace transform variable.

Then,

$$
\begin{gathered}
C(k) \mathbf{q}=\frac{0.5 \bar{s}^{2}+a_{1} \bar{s}+a_{2}}{\bar{s}^{2}+a_{3} \bar{s}+a_{2}} \mathbf{q}=0.5 \mathbf{q}+\frac{a_{4} \bar{s}+0.5 a_{2}}{\bar{s}^{2}+a_{3} \bar{s}+a_{2}} \mathbf{q} \\
C(k) \dot{\mathbf{q}}=\frac{0.5 \bar{s}^{3}+a_{1} \bar{s}^{2}+a_{2} \bar{s}}{\bar{s}^{2}+a_{3} \bar{s}+a_{2}} \frac{2 u}{\bar{c}} \mathbf{q}=0.5 \dot{\mathbf{q}}+a_{4}\left(\frac{2 u}{\bar{c}}\right) \mathbf{q}+\frac{a_{5} \bar{s}+a_{6}}{\bar{s}^{2}+a_{3} \bar{s}+a_{2}}\left(\frac{2 u}{\bar{c}}\right) \mathbf{q}
\end{gathered}
$$

where

$$
\begin{gathered}
a_{4}=a_{1}-0.5 a_{3} \\
a_{5}=0.5 a_{2}-a_{1} a_{3}+0.5 a_{3}^{2} \\
a_{6}=-a_{1} a_{2}+0.5 a_{2} a_{3}
\end{gathered}
$$


The R. T. Jones' unsteady aerodynamic approximation results in the following global matrix equation

$$
\begin{gathered}
\left(\mathbf{M}_{s}+\mathbf{M}_{a n}\right) \ddot{\mathbf{q}}+\mathbf{M}_{e} \ddot{\mathbf{u}}_{e}+\left(\mathbf{C}_{s}+\mathbf{C}_{a n}+0.5 \mathbf{Q}_{a c}\right) \dot{\mathbf{q}}+\left[\mathbf{K}_{s}+0.5 \mathbf{K}_{a c}+a_{4}\left(\frac{2 u}{\bar{c}}\right) \mathbf{C}_{a c}\right] \mathbf{q}+\mathbf{K}_{a c} \mathbf{y}+\left(\frac{2 u}{\bar{c}}\right) \mathbf{C}_{a c} \mathbf{z}=\mathbf{Q}_{e} \dot{\mathbf{x}}_{r} \\
+\mathbf{P}_{e} \mathbf{x}_{r}+\mathbf{F}_{e n} \ddot{\mathbf{u}}_{e}+\left(\mathbf{E}_{e n}+0.5 \mathbf{E}_{e c}\right) \dot{\mathbf{u}}_{e}+\left[\mathbf{D}_{e n}+0.5 \mathbf{D}_{e c}+a_{4}\left(\frac{2 u}{\bar{c}}\right) \mathbf{E}_{e c}\right] \mathbf{u}_{e}+\mathbf{D}_{e c} \mathbf{v}+\left(\frac{2 u}{\bar{c}}\right) \mathbf{E}_{e c} \mathbf{w}+\mathbf{f}_{e g} \quad(202) \\
\ddot{\mathbf{y}}+a_{3}\left(\frac{2 u}{\bar{c}}\right) \dot{\mathbf{y}}+a_{2}\left(\frac{2 u}{\bar{c}}\right)^{2} \mathbf{y}=a_{4}\left(\frac{2 u}{\bar{c}}\right) \dot{\mathbf{q}}+0.5 a_{2}\left(\frac{2 u}{\bar{c}}\right)^{2} \mathbf{q} \\
\ddot{\mathbf{z}}+a_{3}\left(\frac{2 V}{\bar{c}}\right) \dot{\mathbf{z}}+a_{2}\left(\frac{2 V}{\bar{c}}\right)^{2} \mathbf{z}=a_{5}\left(\frac{2 V}{\bar{c}}\right) \dot{\mathbf{q}}+a_{6}\left(\frac{2 V}{\bar{c}}\right)^{2} \mathbf{q} \\
\ddot{\mathbf{v}}+a_{3}\left(\frac{2 u}{\bar{c}}\right) \dot{\mathbf{v}}+a_{2}\left(\frac{2 u}{\bar{c}}\right)^{2} \mathbf{v}=a_{4}\left(\frac{2 u}{\bar{c}}\right) \dot{\mathbf{u}}_{e}+0.5 a_{2}\left(\frac{2 u}{\bar{c}}\right)^{2} \mathbf{u}_{e} \\
\ddot{\mathbf{w}}+a_{3}\left(\frac{2 u}{\bar{c}}\right) \dot{\mathbf{w}}+a_{2}\left(\frac{2 u}{\bar{c}}\right)^{2} \mathbf{w}=a_{5}\left(\frac{2 u}{\bar{c}}\right) \dot{\mathbf{u}}_{e}+a_{6}\left(\frac{2 u}{\bar{c}}\right)^{2} \mathbf{u}_{e}
\end{gathered}
$$

where $\mathbf{y}$ and $\mathbf{z}$ are the unsteady aerodynamic lag state vectors, and $\mathbf{v}$ and $\mathbf{w}$ are the unsteady aerodynamic lag state vectors for the control surfaces.

The nonlinear flight dynamic equations can be cast in a vector form as

$$
\begin{array}{r}
\mathbf{M}_{r r} \dot{\mathbf{x}}_{r}=\mathbf{f}\left(\mathbf{x}_{r}, \dot{\mathbf{x}}_{r}, \mathbf{u}_{r}\right)+\mathbf{V}_{r n} \ddot{\mathbf{q}}+\left[\mathbf{T}_{r n}+C(k) \mathbf{T}_{r c}\right] \dot{\mathbf{q}}+\left[\mathbf{S}_{r n}+C(k) \mathbf{S}_{r c}\right] \mathbf{q}+\mathbf{F}_{r n} \ddot{\mathbf{u}}_{e}+\left[\mathbf{E}_{r n}+C(k) \mathbf{E}_{r c}\right] \dot{\mathbf{u}}_{e} \\
+\left[\mathbf{D}_{r n}+C(k) \mathbf{D}_{r c}\right] \mathbf{u}_{e}+\mathbf{f}_{r g}
\end{array}
$$

where $\mathbf{u}_{r}=\left[\begin{array}{ll}\delta_{e} & \delta_{r}\end{array}\right]^{\top}$ is the rigid-body aircraft control vector with $\delta_{e}$ as the elevator deflection and $\delta_{r}$ as the rudder deflection, and $\mathbf{f}_{r g}$ is the vector of the aerodynamic forces and moments due to the gust.

The nonlinear flight dynamic equations can be linearized and separated into longitudinal dynamics and lateraldirectional dynamics. The equations for the aircraft position $x$ and $y$ are uncoupled to the other equations. The longitudinal dynamics include the rigid-body aircraft state vector $\mathbf{x}_{r}=\left[\begin{array}{lllll}h & u & w & q & \theta\end{array}\right]^{\top}$ and the lateral-directional dynamics include the rigid-body aircraft state vector $\mathbf{x}_{r}=\left[\begin{array}{lllll}v & p & r & \phi & \psi\end{array}\right]^{\top}$. The linearized equations of motion can be expressed as

$$
\begin{aligned}
\mathbf{M}_{r} \dot{\mathbf{x}}_{r}=\mathbf{Q}_{r} \dot{\mathbf{x}}_{r}+ & \mathbf{P}_{r} \mathbf{x}_{r}+\mathbf{D}_{r} \mathbf{u}_{r}+\mathbf{V}_{r n} \ddot{\mathbf{q}}+\left(\mathbf{T}_{r n}+0.5 \mathbf{T}_{r c}\right) \dot{\mathbf{q}}+\left[\mathbf{S}_{r n}+0.5 \mathbf{S}_{r c}+a_{4}\left(\frac{2 u}{\bar{c}}\right) \mathbf{T}_{r c}\right] \mathbf{q}+\mathbf{S}_{r c} \mathbf{y}+\left(\frac{2 u}{\bar{c}}\right) \mathbf{T}_{r c} \mathbf{z} \\
& +\mathbf{F}_{r n} \ddot{\mathbf{u}}_{e}+\left(\mathbf{E}_{r n}+0.5 \mathbf{E}_{r c}\right) \dot{\mathbf{u}}_{e}+\left[\mathbf{D}_{r n}+0.5 \mathbf{D}_{r c}+a_{4}\left(\frac{2 u}{\bar{c}}\right) \mathbf{E}_{r c}\right] \mathbf{u}_{e}+\mathbf{D}_{r c} \mathbf{v}+\left(\frac{2 u}{\bar{c}}\right) \mathbf{E}_{r c} \mathbf{w}+\mathbf{f}_{r g} \quad(208
\end{aligned}
$$

The actuator dynamic equation of the rigid-body aircraft control is assumed to be a first-order model

$$
\dot{\mathbf{u}}_{r}=-\Lambda\left(\mathbf{u}_{r}-\mathbf{u}_{r c}\right)
$$

where $\Lambda=\Lambda^{\top}>0$ is the actuator rate vector and $\mathbf{u}_{r c}$ is the command vector of the rigid-body aircraft control.

The control surfaces contribute to both the rigid-body aircraft flight dynamics and wing aeroelasticity. The dynamics of the control surfaces are governed by the mass, damping, and stiffness of the control surfaces and their backup structures and the unsteady aerodynamic hinge moment. The contributions to the unsteady aerodynamic hinge moment of a control surface come from the control surface deflection and its velocity and acceleration, rigid-body aircraft states, and the wing elastic states. 
The general equation of motion of the control surfaces can be written as

$$
\begin{aligned}
\mathbf{M}_{\delta} \ddot{\mathbf{u}}_{e}+ & \mathbf{M}_{e} \ddot{\mathbf{q}}+\mathbf{C}_{\delta} \dot{\mathbf{u}}_{e}+\mathbf{K}_{\delta} \mathbf{u}_{e}=\mathbf{Q}_{\delta} \dot{\mathbf{x}}_{r}+\mathbf{P}_{\delta} \mathbf{x}_{r}+\mathbf{V}_{\delta n} \ddot{\mathbf{q}}+\left(\mathbf{T}_{\delta n}+0.5 \mathbf{T}_{\delta c}\right) \dot{\mathbf{q}}+\left[\mathbf{S}_{\delta n}+0.5 \mathbf{S}_{\delta c}+a_{4}\left(\frac{2 u}{\bar{c}}\right) \mathbf{T}_{\delta c}\right] \mathbf{q} \\
& +\mathbf{S}_{\delta c} \mathbf{y}+\left(\frac{2 u}{\bar{c}}\right) \mathbf{T}_{\delta c} \mathbf{z}+\mathbf{F}_{\delta n} \ddot{\mathbf{u}}_{e}+\left(\mathbf{E}_{\delta n}+0.5 \mathbf{E}_{\delta c}\right) \dot{\mathbf{u}}_{e}+\left[\mathbf{D}_{\delta n}+0.5 \mathbf{D}_{\delta c}+a_{4}\left(\frac{2 u}{\bar{c}}\right) \mathbf{E}_{\delta c}\right] \mathbf{u}_{e}+\mathbf{D}_{\delta c} \mathbf{v} \\
& +\left(\frac{2 u}{\bar{c}}\right) \mathbf{E}_{\delta c} \mathbf{w}+\tau_{\delta}
\end{aligned}
$$

where $\tau_{\delta}$ is the control torque vector.

The servo-dynamic equation for the control surfaces is based on a proportional-integral-derivative (PID) feedback control law as follows:

$$
\begin{gathered}
\dot{\mathbf{e}}=\mathbf{u}_{e}-\mathbf{u}_{e c} \\
\tau_{\delta}=\mathbf{k}_{p}\left(\mathbf{u}_{e}-\mathbf{u}_{e c}\right)+\mathbf{k}_{i} \mathbf{e}+\mathbf{k}_{d} \dot{\mathbf{u}}_{e}
\end{gathered}
$$

where $\mathbf{e}$ is the integral error of the aeroelastic control command $\mathbf{u}_{e c}$.

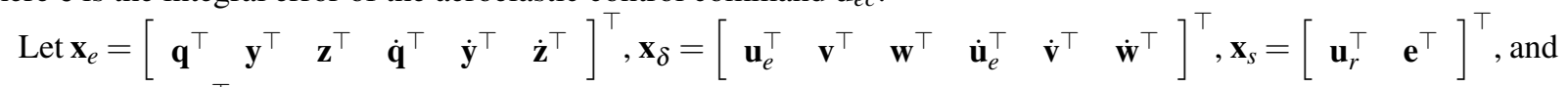
$\mathbf{u}=\left[\begin{array}{ll}\mathbf{u}_{r c}^{\top} & \mathbf{u}_{e c}^{\top}\end{array}\right]^{\top}$. Then, the integrated ASE flight dynamic equation can be expressed in the state space form

$$
\dot{\mathbf{x}}=\mathbf{A x}+\mathbf{B u}+\mathbf{w}_{g}
$$

where $\mathbf{x}=\left[\begin{array}{llll}\mathbf{x}_{r}^{\top} & \mathbf{x}_{e}^{\top} & \mathbf{x}_{\delta}^{\top} & \mathbf{x}_{s}^{\top}\end{array}\right]^{\top}, \mathbf{A}=\mathbf{M}^{-1} \mathbf{S}, \mathbf{B}=\mathbf{M}^{-1} \mathbf{T}$, and $\mathbf{w}_{g}=\mathbf{M}^{-1} \mathbf{F}$.

The mass, stiffness, and control force matrices $\mathbf{M}, \mathbf{S}$, and $\mathbf{T}$ are given by

$\mathbf{M}=\left[\begin{array}{c|cccccc|cccccc|cc}\mathbf{M}_{r}-\mathbf{Q}_{r} & \mathbf{0} & \mathbf{0} & \mathbf{0} & -\mathbf{V}_{r n} & \mathbf{0} & \mathbf{0} & \mathbf{0} & \mathbf{0} & \mathbf{0} & -\mathbf{F}_{r n} & \mathbf{0} & \mathbf{0} & \mathbf{0} & \mathbf{0} \\ \hline \mathbf{0} & \mathbf{I} & \mathbf{0} & \mathbf{0} & \mathbf{0} & \mathbf{0} & \mathbf{0} & \mathbf{0} & \mathbf{0} & \mathbf{0} & \mathbf{0} & \mathbf{0} & \mathbf{0} & \mathbf{0} & \mathbf{0} \\ \mathbf{0} & \mathbf{0} & \mathbf{I} & \mathbf{0} & \mathbf{0} & \mathbf{0} & \mathbf{0} & \mathbf{0} & \mathbf{0} & \mathbf{0} & \mathbf{0} & \mathbf{0} & \mathbf{0} & \mathbf{0} & \mathbf{0} \\ \mathbf{0} & \mathbf{0} & \mathbf{0} & \mathbf{I} & \mathbf{0} & \mathbf{0} & \mathbf{0} & \mathbf{0} & \mathbf{0} & \mathbf{0} & \mathbf{0} & \mathbf{0} & \mathbf{0} & \mathbf{0} & \mathbf{0} \\ -\mathbf{Q}_{e} & \mathbf{0} & \mathbf{0} & \mathbf{0} & \mathbf{M}_{s}+\mathbf{M}_{a n} & \mathbf{0} & \mathbf{0} & \mathbf{0} & \mathbf{0} & \mathbf{0} & \mathbf{M}_{e}-\mathbf{F}_{e n} & \mathbf{0} & \mathbf{0} & \mathbf{0} & \mathbf{0} \\ \mathbf{0} & \mathbf{0} & \mathbf{0} & \mathbf{0} & \mathbf{0} & \mathbf{I} & \mathbf{0} & \mathbf{0} & \mathbf{0} & \mathbf{0} & \mathbf{0} & \mathbf{0} & \mathbf{0} & \mathbf{0} & \mathbf{0} \\ \mathbf{0} & \mathbf{0} & \mathbf{0} & \mathbf{0} & \mathbf{0} & \mathbf{0} & \mathbf{I} & \mathbf{0} & \mathbf{0} & \mathbf{0} & \mathbf{0} & \mathbf{0} & \mathbf{0} & \mathbf{0} & \mathbf{0} \\ \hline \mathbf{0} & \mathbf{0} & \mathbf{0} & \mathbf{0} & \mathbf{0} & \mathbf{0} & \mathbf{0} & \mathbf{I} & \mathbf{0} & \mathbf{0} & \mathbf{0} & \mathbf{0} & \mathbf{0} & \mathbf{0} & \mathbf{0} \\ \mathbf{0} & \mathbf{0} & \mathbf{0} & \mathbf{0} & \mathbf{0} & \mathbf{0} & \mathbf{0} & \mathbf{0} & \mathbf{I} & \mathbf{0} & \mathbf{0} & \mathbf{0} & \mathbf{0} & \mathbf{0} & \mathbf{0} \\ \mathbf{0} & \mathbf{0} & \mathbf{0} & \mathbf{0} & \mathbf{0} & \mathbf{0} & \mathbf{0} & \mathbf{0} & \mathbf{0} & \mathbf{I} & \mathbf{0} & \mathbf{0} & \mathbf{0} & \mathbf{0} & \mathbf{0} \\ -\mathbf{Q}_{\delta} & \mathbf{0} & \mathbf{0} & \mathbf{0} & \mathbf{M}_{e}-\mathbf{V}_{\delta n} & \mathbf{0} & \mathbf{0} & \mathbf{0} & \mathbf{0} & \mathbf{0} & \mathbf{M}_{\delta}-\mathbf{F}_{\delta n} & \mathbf{0} & \mathbf{0} & \mathbf{0} & \mathbf{0} \\ \mathbf{0} & \mathbf{0} & \mathbf{0} & \mathbf{0} & \mathbf{0} & \mathbf{0} & \mathbf{0} & \mathbf{0} & \mathbf{0} & \mathbf{0} & \mathbf{0} & \mathbf{I} & \mathbf{0} & \mathbf{0} & \mathbf{0} \\ \mathbf{0} & \mathbf{0} & \mathbf{0} & \mathbf{0} & \mathbf{0} & \mathbf{0} & \mathbf{0} & \mathbf{0} & \mathbf{0} & \mathbf{0} & \mathbf{0} & \mathbf{0} & \mathbf{I} & \mathbf{0} & \mathbf{0} \\ \hline \mathbf{0} & \mathbf{0} & \mathbf{0} & \mathbf{0} & \mathbf{0} & \mathbf{0} & \mathbf{0} & \mathbf{0} & \mathbf{0} & \mathbf{0} & \mathbf{0} & \mathbf{0} & \mathbf{0} & \mathbf{I} & \mathbf{0} \\ \mathbf{0} & \mathbf{0} & \mathbf{0} & \mathbf{0} & \mathbf{0} & \mathbf{0} & \mathbf{0} & \mathbf{0} & \mathbf{0} & \mathbf{0} & \mathbf{0} & \mathbf{0} & \mathbf{0} & \mathbf{0} & \mathbf{I}\end{array}\right]$




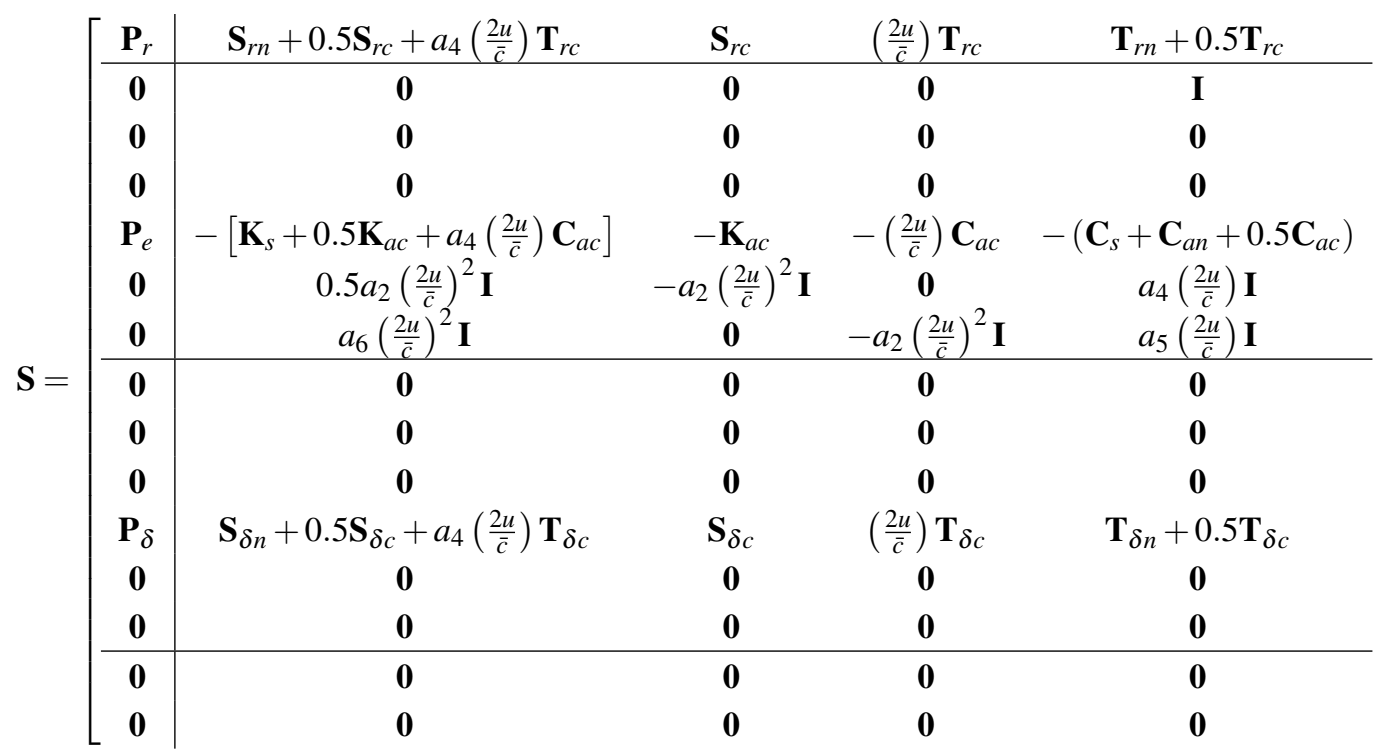

\begin{tabular}{cc|ccc}
$\mathbf{0}$ & $\mathbf{0}$ & $\mathbf{D}_{r n}+0.5 \mathbf{D}_{r c}+a_{4}\left(\frac{2 u}{\bar{c}}\right) \mathbf{E}_{r c}$ & $\mathbf{D}_{r c}$ & $\left(\frac{2 u}{\bar{c}}\right) \mathbf{E}_{r c}$ \\
\hline $\mathbf{0}$ & $\mathbf{0}$ & $\mathbf{0}$ & $\mathbf{0}$ & $\mathbf{0}$ \\
$\mathbf{I}$ & $\mathbf{0}$ & $\mathbf{0}$ & $\mathbf{0}$ & $\mathbf{0}$ \\
$\mathbf{0}$ & $\mathbf{I}$ & $\mathbf{0}$ & $\mathbf{0}$ & $\mathbf{0}$ \\
$\mathbf{0}$ & $\mathbf{0}$ & $\mathbf{D}_{e n}+0.5 \mathbf{D}_{e c}+a_{4}\left(\frac{2 u}{\bar{c}}\right) \mathbf{E}_{e c}$ & $\mathbf{D}_{e c}$ & $\left(\frac{2 u}{\bar{c}}\right) \mathbf{E}_{e c}$ \\
$-a_{3}\left(\frac{2 u}{\bar{c}}\right) \mathbf{I}$ & $\mathbf{0}$ & $\mathbf{0}$ & $\mathbf{0}$ & $\mathbf{0}$ \\
$\mathbf{0}$ & $-a_{3}\left(\frac{2 u}{\bar{c}}\right) \mathbf{I}$ & $\mathbf{0}$ & $\mathbf{0}$ & $\mathbf{0}$ \\
\hline $\mathbf{0}$ & $\mathbf{0}$ & $\mathbf{0}$ & $\mathbf{0}$ & $\mathbf{0}$ \\
$\mathbf{0}$ & $\mathbf{0}$ & $\mathbf{0}$ & $\mathbf{0}$ & $\mathbf{0}$ \\
$\mathbf{0}$ & $\mathbf{0}$ & $\mathbf{0}$ & $\mathbf{0}$ \\
$\mathbf{0}$ & $\mathbf{0}$ & $-\mathbf{K}_{\delta}+\mathbf{D}_{\delta n}+0.5 \mathbf{D}_{\delta c}+a_{4}\left(\frac{2 u}{\bar{c}}\right) \mathbf{E}_{\delta c}+\mathbf{k}_{p}$ & $\mathbf{D}_{\delta c}$ & $\left(\frac{2 u}{\bar{c}}\right) \mathbf{E}_{\delta c}$ \\
$\mathbf{0}$ & $\mathbf{0}$ & $0.5 a_{2}\left(\frac{2 u}{\bar{c}}\right)^{2} \mathbf{I}$ & $-a_{2}\left(\frac{2 u}{\bar{c}}\right)^{2} \mathbf{I}$ & $\mathbf{0}$ \\
$\mathbf{0}$ & $\mathbf{0}$ & $a_{6}\left(\frac{2 u}{\bar{c}}\right)^{2} \mathbf{I}$ & $\mathbf{0}$ & $-a_{2}\left(\frac{2 u}{\bar{c}}\right)^{2} \mathbf{I}$ \\
\hline $\mathbf{0}$ & $\mathbf{0}$ & $\mathbf{0}$ & $\mathbf{0}$ & $\mathbf{0}$ \\
$\mathbf{0}$ & $\mathbf{0}$ & $\mathbf{I}$ & $\mathbf{0}$ & $\mathbf{0}$
\end{tabular}

$\left.\begin{array}{ccc|cc}\mathbf{E}_{r n}+0.5 \mathbf{E}_{r c} & \mathbf{0} & \mathbf{0} & \mathbf{D}_{r} & \mathbf{0} \\ \hline \mathbf{0} & \mathbf{0} & \mathbf{0} & \mathbf{0} & \mathbf{0} \\ \mathbf{0} & \mathbf{0} & \mathbf{0} & \mathbf{0} & \mathbf{0} \\ \mathbf{0} & \mathbf{0} & \mathbf{0} & \mathbf{0} & \mathbf{0} \\ \mathbf{E}_{e n}+0.5 \mathbf{E}_{e c} & \mathbf{0} & \mathbf{0} & \mathbf{0} & \mathbf{0} \\ \mathbf{0} & \mathbf{0} & \mathbf{0} & \mathbf{0} & \mathbf{0} \\ \mathbf{0} & \mathbf{0} & \mathbf{0} & \mathbf{0} & \mathbf{0} \\ \hline \mathbf{I} & \mathbf{0} & \mathbf{0} & \mathbf{0} & \mathbf{0} \\ \mathbf{0} & \mathbf{I} & \mathbf{0} & \mathbf{0} & \mathbf{0} \\ \mathbf{0} & \mathbf{0} & \mathbf{I} & \mathbf{0} & \mathbf{0} \\ -\mathbf{C}_{\delta}+\mathbf{E}_{\delta n}+0.5 \mathbf{E}_{\delta c}+\mathbf{k}_{d} & \mathbf{0} & \mathbf{0} & \mathbf{0} & \mathbf{k}_{i} \\ a_{4}\left(\frac{2 u}{\bar{c}}\right) \mathbf{I} & -a_{3}\left(\frac{2 u}{\bar{c}}\right) \mathbf{I} & \mathbf{0} & \mathbf{0} & \mathbf{0} \\ a_{5}\left(\frac{2 u}{\bar{c}}\right) \mathbf{I} & \mathbf{0} & -a_{3}\left(\frac{2 u}{\bar{c}}\right) \mathbf{I} & \mathbf{0} & \mathbf{0} \\ \hline \mathbf{0} & \mathbf{0} & \mathbf{0} & -\Lambda & \mathbf{0} \\ \mathbf{0} & \mathbf{0} & \mathbf{0} & \mathbf{0} & \mathbf{0}\end{array}\right]$




$$
\begin{gathered}
\mathbf{T}=\left[\begin{array}{cc}
\mathbf{0} & \mathbf{0} \\
\hline \mathbf{0} & \mathbf{0} \\
\mathbf{0} & \mathbf{0} \\
\mathbf{0} & \mathbf{0} \\
\mathbf{0} & \mathbf{0} \\
\mathbf{0} & \mathbf{0} \\
\mathbf{0} & \mathbf{0} \\
\hline \mathbf{0} & \mathbf{0} \\
\mathbf{0} & \mathbf{0} \\
\mathbf{0} & \mathbf{0} \\
\mathbf{0} & -\mathbf{k}_{p} \\
\mathbf{0} & \mathbf{0} \\
\mathbf{0} & \mathbf{0} \\
\hline \Lambda & \mathbf{0} \\
\mathbf{0} & -\mathbf{I}
\end{array}\right] \\
\mathbf{F}=\left[\begin{array}{c}
\mathbf{f}_{r g} \\
\hline \mathbf{0} \\
\mathbf{0} \\
\mathbf{0} \\
\mathbf{f}_{e g} \\
\mathbf{0} \\
\mathbf{0} \\
\hline \mathbf{0} \\
\mathbf{0} \\
\mathbf{0} \\
\mathbf{0} \\
\mathbf{0} \\
\mathbf{0} \\
\hline 0 \\
\mathbf{0}
\end{array}\right]
\end{gathered}
$$

The output is assumed to be an acceleration measurement at a given location $\left(x_{c}, y_{c}, z_{c}\right)$ on the wing which is computed as

$$
\ddot{z}_{a}=W_{t t}+y_{c} \Theta_{t t}=\Phi \ddot{\mathbf{q}}
$$

where $\Phi$ is a modal transformation vector.

Then,

$$
\ddot{z}_{a}=\Phi \mathbf{I}_{\ddot{q}} \dot{\mathbf{x}}=\Phi \mathbf{I}_{\ddot{q}}\left(\mathbf{A} \mathbf{x}+\mathbf{B u}+\mathbf{w}_{g}\right)
$$

where $\mathbf{I}_{\ddot{q}}=\left[\begin{array}{lllllllllllllll}\mathbf{0} & \mathbf{0} & \mathbf{0} & \mathbf{0} & \mathbf{I} & \mathbf{0} & \mathbf{0} & \mathbf{0} & \mathbf{0} & \mathbf{0} & \mathbf{0} & \mathbf{0} & \mathbf{0} & \mathbf{0} & \mathbf{0}\end{array}\right]$ such that $\ddot{\mathbf{q}}=\mathbf{I}_{\ddot{q}} \dot{\mathbf{x}}$.

Let $y=\ddot{z}_{a}$ be the output, then

$$
y=\mathbf{C x}+\mathbf{D u}+\mathbf{E} \mathbf{w}_{g}
$$

where $\mathbf{C}=\Phi \mathbf{I}_{\ddot{q}} \mathbf{A}$ and $\mathbf{D}=\Phi \mathbf{I}_{\ddot{q}} \mathbf{B}$.

\section{Simulations}

A finite-element model (FEM) of the flexible wing is constructed using 33 elements. The FEM computes the mode shapes and displacements of the wing. The generalized coordinates are constructed from the FEM and are coupled to the aircraft nonlinear flight dynamics. 
The flexible wing GTM simulations are conducted at Mach 0.797 and 36,000 ft with $80 \%$ fuel loading. Figures 9 and 10 show the aeroservoelastic (ASE) pole locations of the first 198 symmetric and anti-symmetric modes of the flexible wing GTM. All the ASE poles are stable at this flight condition.
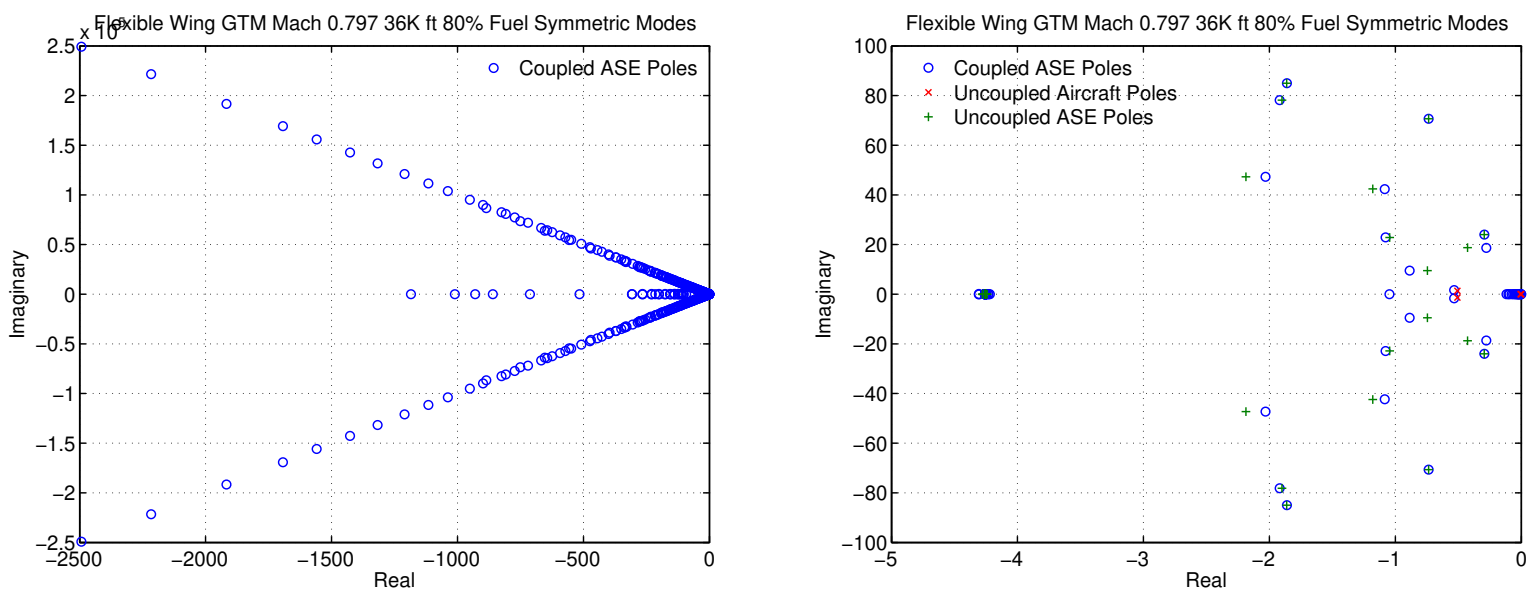

Figure 9. Pole Locations of Symmetric Modes of Flexible Wing GTM
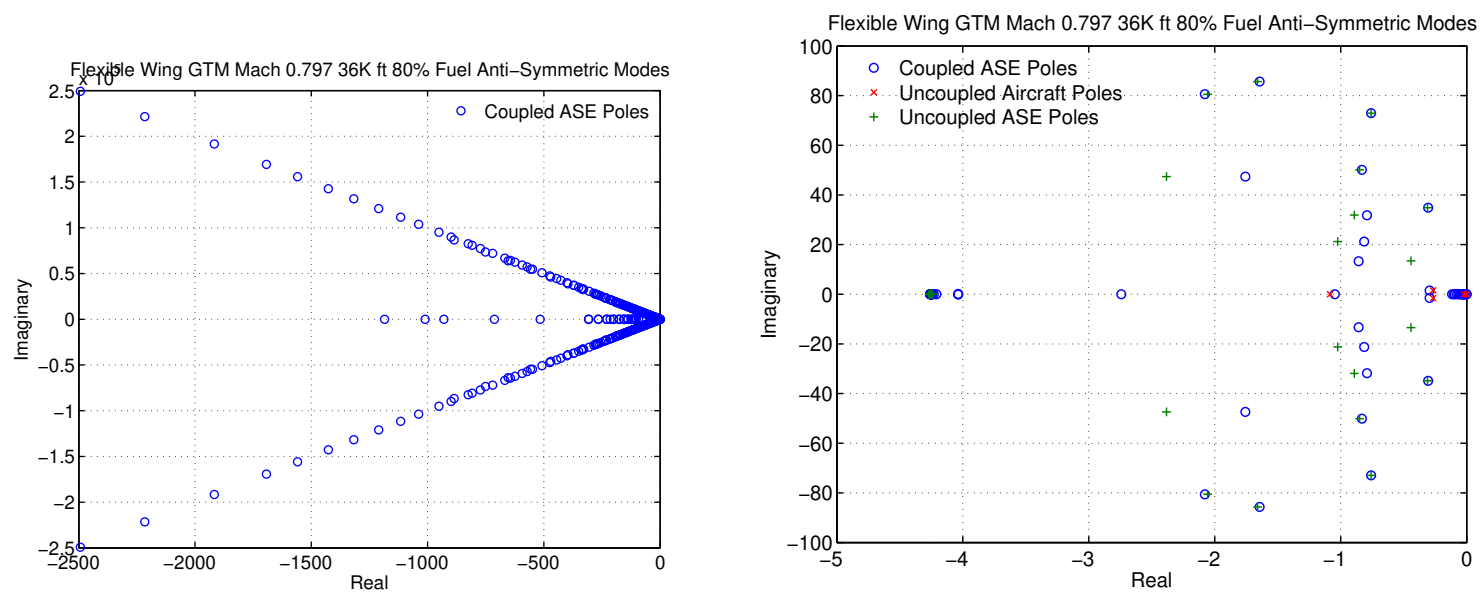

Figure 10. Pole Locations of Anti-Symmetric Modes of Flexible Wing GTM

Table 1 shows the eigenvalues of the ASE coupled and uncoupled rigid-body aircraft modes of the GTM. The ASE coupling does not seem to significantly alter the rigid-body aircraft modes.

Table 2 shows the eigenvalues of the symmetric and anti-symmetric ASE modes of the flexible GTM wing. The fundamental frequency of the first bending symmetric ASE mode is $9.4109 \mathrm{rad} / \mathrm{sec}$ or $1.4978 \mathrm{~Hz}$. The fundamental frequency of the first bending anti-symmetric ASE mode is $13.2072 \mathrm{rad} / \mathrm{sec}$ or $2.1020 \mathrm{~Hz}$. A flutter analysis is conducted and shows that the first flutter mode is associated with the second bending symmetric ASE mode which occurs at Mach 0.958 corresponding to a flutter frequency of $17.8167 \mathrm{rad} / \mathrm{sec}$ or $2.8356 \mathrm{~Hz}$. 


\begin{tabular}{|c|c|c|}
\hline $\begin{array}{c}\text { Rigid-Body } \\
\text { Mode }\end{array}$ & $\begin{array}{c}\text { ASE Coupled } \\
\text { Eigenvalues }\end{array}$ & $\begin{array}{c}\text { Uncoupled } \\
\text { Eigenvalues }\end{array}$ \\
\hline \hline Phugoid & $-0.0064 \pm 0.0837 i$ & $-0.0047 \pm 0.0828 i$ \\
\hline \hline Short Period & $-0.5328 \pm 1.6576 i$ & $-0.5071 \pm 1.3590 i$ \\
\hline Dutch-Roll & $-0.2939 \pm 1.5059 i$ & $-0.2663 \pm 1.5492 i$ \\
\hline Roll & -1.0472 & -1.0845 \\
\hline Spiral & -0.0202 & -0.0219 \\
\hline
\end{tabular}

Table 1. Eigenvalues of ASE Coupled and Uncoupled Rigid-Body Modes of the Flexible Wing GTM

\begin{tabular}{|c|c|c|c|c|}
\hline $\begin{array}{c}\text { ASE } \\
\text { Mode }\end{array}$ & $\begin{array}{c}\text { Symmetric ASE Mode } \\
\text { Eigenvalues }\end{array}$ & $\begin{array}{c}\text { Symmetric Structural } \\
\text { Dynamic Mode } \\
\text { Eigenvalues }\end{array}$ & $\begin{array}{c}\text { Anti-Symmetric ASE } \\
\text { Mode Eigenvalues }\end{array}$ & $\begin{array}{c}\text { Anti-Symmetric } \\
\text { Structural Dynamic } \\
\text { Mode Eigenvalues }\end{array}$ \\
\hline \hline 1 & $-0.8426 \pm 9.3731 i$ & $-0.0729 \pm 7.2936 i$ & $-0.8009 \pm 13.1829 i$ & $-0.1116 \pm 11.1569 i$ \\
\hline 2 & $-0.2818 \pm 18.6759 i$ & $-0.1907 \pm 19.0694 i$ & $-0.7932 \pm 21.2460 i$ & $-0.2107 \pm 21.0691 i$ \\
\hline 3 & $-1.0351 \pm 22.8182 i$ & $-0.2153 \pm 21.5297 i$ & $-0.7716 \pm 31.7682 i$ & $-0.3117 \pm 31.1694 i$ \\
\hline 4 & $-0.2903 \pm 24.0005 i$ & $-0.2401 \pm 24.0042 i$ & $-0.3090 \pm 34.8691 i$ & $-0.3485 \pm 34.8473 i$ \\
\hline 5 & $-1.0543 \pm 42.3144 i$ & $-0.4192 \pm 41.9200 i$ & $-1.7224 \pm 47.5924 i$ & $-0.4991 \pm 49.9050 i$ \\
\hline 6 & $-1.9655 \pm 47.5078 i$ & $-0.5160 \pm 51.5980 i$ & $-0.8172 \pm 50.1101 i$ & $-0.5174 \pm 51.7423 i$ \\
\hline 7 & $-0.7339 \pm 70.6620 i$ & $-0.7065 \pm 70.6513 i$ & $-0.7575 \pm 72.9492 i$ & $-0.7294 \pm 72.9370 i$ \\
\hline 8 & $-1.8622 \pm 78.2028 i$ & $-0.7900 \pm 78.9950 i$ & $-2.0143 \pm 80.6593 i$ & $-0.8188 \pm 81.8763 i$ \\
\hline 9 & $-1.8256 \pm 85.0657 i$ & $-0.8735 \pm 87.3478 i$ & $-1.6216 \pm 85.7461 i$ & $-0.8760 \pm 87.5923 i$ \\
\hline 10 & $-2.3754 \pm 122.32 i$ & $-1.2318 \pm 123.17 i$ & $-2.3030 \pm 128.34 i$ & $-1.2332 \pm 123.31 i$ \\
\hline
\end{tabular}

Table 2. Eigenvalues of ASE and Structural Dynamic Modes of the GTM Flexible Wing

Three types of gust models are incorporated in the ASE flight dynamic simulations of the GTM: a discrete oneminus-cosine gust model, and two continuous gust models based on the Dryden turbulence model and the von Karman turbulence model. Three types of simulations are performed: linear flight dynamics with linear ASE by neglecting the coupling of the rigid-body aircraft states in the aeroelastic partial derivatives, nonlinear flight dynamics with linear ASE, and nonlinear flight dynamics with nonlinear ASE due to the coupling of the rigid-body aircraft states in the aeroelastic partial derivatives. These simulations do not include the coupling of the coupling of the rigid-body aircraft states in the inertial forces and moment.

Figure 11 shows the longitudinal response of the flexible wing GTM due to a doublet elevator input with an amplitude of $1^{\circ}$ under a discrete gust load with a gust amplitude of $10 \mathrm{ft} / \mathrm{sec}$ which represents a moderate gust. The aircraft angle of attack and pitch angle are shown in Figure 11. Both the nonlinear and linear flight dynamics with linear ASE produce aircraft responses that are in very good agreement with each other. However, the nonlinear flight dynamics with nonlinear ASE shows a significant discrepancy in the aircraft response particularly in wing tip bending deflection. This discrepancy illustrates the influence of nonlinear ASE on flexible aircraft flight dynamics due to the coupling of the rigid-body aircraft states with aeroelasticity.

Figure 12 shows the longitudinal response of the flexible wing GTM due to a doublet elevator input under a continuous Dryden gust load with a moderate gust amplitude. The aircraft response with the nonlinear flight dynamics and nonlinear ASE also exhibit differences from the responses with the other two simulation models as seen with the discrete gust simulations. 

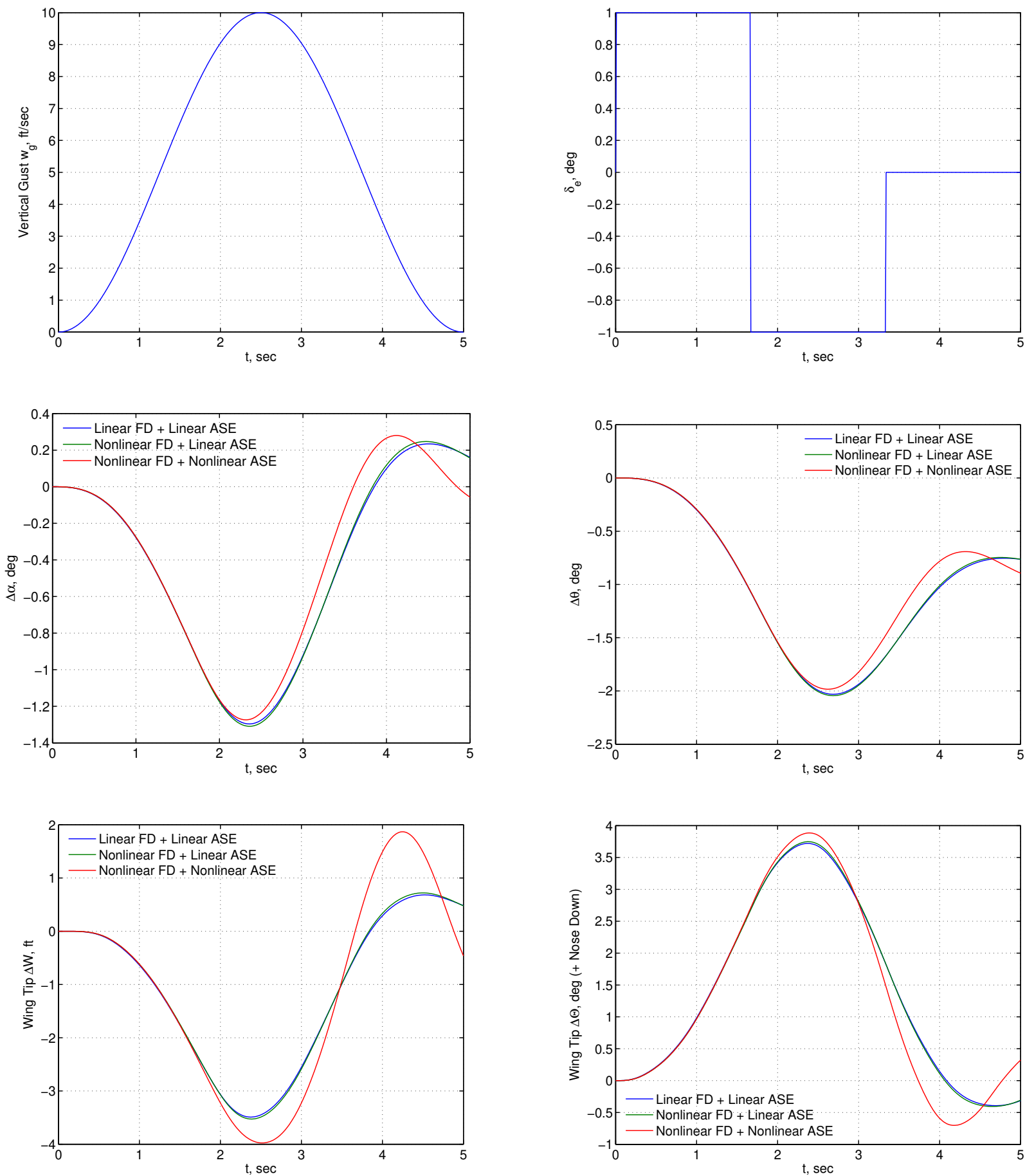

Figure 11. Longitudinal Response of Flexible Wing GTM with 1-Cosine Discrete Gust 

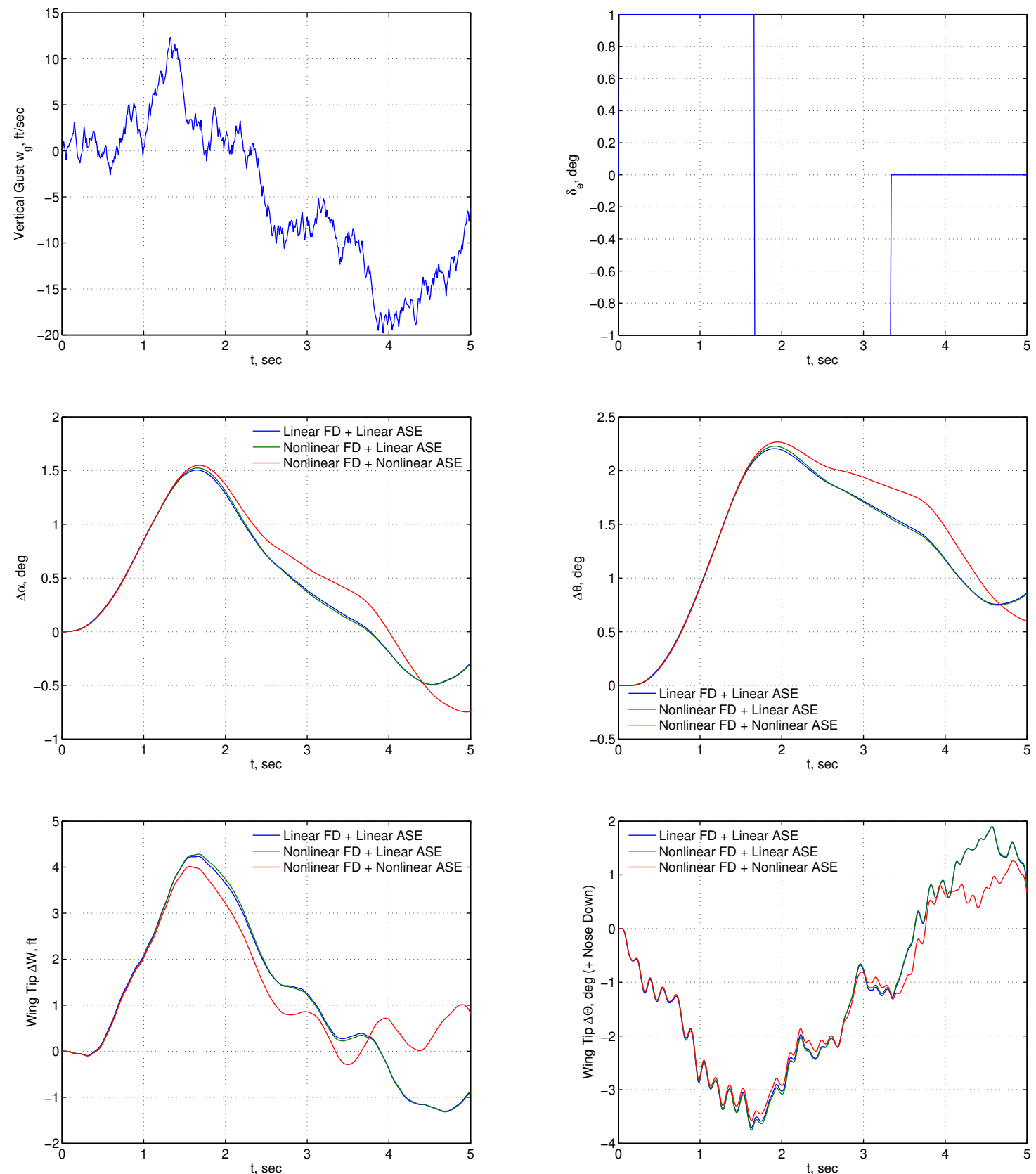

Figure 12. Longitudinal Response of Flexible Wing GTM with Dryden Gust

\section{Conclusions}

This paper describes the development of an integrated nonlinear aeroservoelastic flight dynamic model of the NASA Generic Transport Model (GTM) equipped with flexible wings. The integrated ASE model provides the capability for flight control development and simulations of a highly flexible wing transport aircraft equipped with a new type of distributed flight control surfaces called the Variable Camber Continuous Trailing Edge Flap (VCCTEF). The flexible wing is modeled to be half as stiff as a conventional wing to achieve a wing tip deflection of about $10 \%$ of the 
wing semi-span. The nonlinear aeroservoelasticity in the modeling approach is due to the coupling of the rigid-body aircraft states in the inertial forces and moment as well as in the aeroelastic angle of attack. The unsteady aerodynamics is modeled using the Theodorsen's theory and is corrected for the transonic flow and viscous effect using a transonic small disturbance and integral boundary layer correction method. To construct the aeroservoelastic model, the R. T. Jones method is used to approximate the Theodorsen's function. A proportional-integral-derivative control law is implemented for the flight control surfaces in the integrated aeroservoelastic model. Gust models include the discrete one-minus cosine gust model and the continuous Dryden and von Karman turbulence models.

Simulations of the flexible wing GTM at Mach 0.797 and 36,000 ft with $80 \%$ fuel loading are conducted. The simulations show that the rigid-body aircraft modes are not significantly influenced by the aeroservoelastic coupling. Three types of simulations are performed: linear flight dynamics with linear aeroelasticity by neglecting the coupling of the rigid-body aircraft states in the aeroelastic partial derivatives, nonlinear flight dynamics with linear aeroservoelasticity, and nonlinear flight dynamics with nonlinear aeroservoelasticity due to the coupling of the rigid-body aircraft states in the aeroelastic partial derivatives. The aircraft responses to the one-minus cosine gust and the continuous Dryden gust show that the nonlinear aeroservoelastic model produces significantly different responses than both linear aeroservoelastic models. Future work will further include the nonlinear aeroelasticity due to the coupling of the rigid-body aircraft states in the inertial forces and moment acting on the wing.

\section{References}

\footnotetext{
${ }^{1}$ Jordan, T. L., Langford, W. M., Belcastro, C. M., Foster, J. M., Shah, G. H., Howland, G., and Kidd, R., "Development of a Dynamically Scaled Generic Transport Model Testbed for Flight Research Experiments," AUVSI Unmanned Unlimited, Arlington, VA, 2004.

${ }^{2}$ Nguyen, N., "Elastically Shaped Future Air Vehicle Concept," NASA Innovation Fund Award 2010 Report, October 2010, Submitted to NASA Innovative Partnerships Program.

${ }^{3}$ Nguyen, N., Trinh, K., Reynolds, K., Kless, J., Aftosmis, M., Urnes, J., and Ippolito, C., "Elastically Shaped Wing Optimization and Aircraft Concept for Improved Cruise Efficiency," AIAA Aerospace Sciences Meeting, AIAA-2013-0141, January 2013.

${ }^{4}$ Boeing Report No. 2012X0015, "Development of Variable Camber Continuous Trailing Edge Flap System," October 4, 2012.

${ }^{5}$ Urnes, J., Nguyen, N., Ippolito, C., Totah, J., Trinh, K., and Ting, E., “A Mission Adaptive Variable Camber Flap Control System to Optimize High Lift and Cruise Lift to Drag Ratios of Future N+3 Transport Aircraft," AIAA Aerospace Sciences Meeting, AIAA-2013-0214, January 2013.

${ }^{6}$ Boeing Report No. 2014X0030, "Development of Variable Camber Continuous Trailing Edge Flap System for B757 Configured with a More Flexible Wing," Submitted to NASA, September 27, 2014.

${ }^{7}$ Nguyen, N., Precup, N., Urnes, J., Nelson, C., Lebofsky, S., Ting, E., and Livne, E., "Experimental Investigation of a Flexible Wing with a Variable Camber Continuous Trailing Edge Flap Design," 32nd AIAA Applied Aerodynamics, AIAA 2014-2441, June 2014.

${ }^{8}$ Nguyen, N. T., Precup, N., Livne, L., Urnes, J., Dickey, E., Nelson, C., Chiew, J., Rodriguez, D., Ting, E., and Lebofsky, S., "Wind Tunnel Investigation of a Flexible Wing High-Lift Configuration with a Variable Camber Continuous Trailing Edge Flap Design," 33rd AIAA Applied Aerodynamics Confererence, AIAA-2015-2417, June 2015.

${ }^{9}$ Chaparro, D., Fujiwara, G. E., Ting, E., and Nguyen, N. T., "Transonic and Viscous Models for Vortex Lattice Method Applied to Transport Aircraft," 35th AIAA Applied Aerodynamics Conference, AIAA-2017-4221, June 2017.

${ }^{10}$ Houbolt, J. C. and Brooks, G. W., "Differential Equations of Motion for Combined Flapwise Bending, Chordwise Bending, and Torsion of Twisted Nonuniform Rotor Blades," NACA Technical Note 3905, February 1957.

${ }^{11}$ Nguyen, N., "Integrated Flight Dynamics Modeling of Flexible Aircraft with Inertial Force-Propulsion - Aeroelastic Couplings," 46th AIAA Aerospace Sciences Meeting and Exhibit, AIAA-2008-0194, Reno, NV, January 2008.

${ }^{12}$ Nguyen, N. and Ting, E., "Inertial Force Coupling to Nonlinear Aeroelasticity of Flexible Wing Aircraft," AIAA Dynamics Specialists Conference, AIAA-2016-1094, January 2016.

${ }^{13}$ Theodorsen, T., "General Theory of Aerodynamic Instability and the Mechanism of Flutter," NACA Report 496, 1934.

${ }^{14}$ Chaparro, D., Fujiwara, G. E., Ting, E., and Nguyen, N. T., "Transonic and Viscous Potential Flow Method Applied to Flexible Wing Transport Aircraft," 35th AIAA Applied Aerodynamics Conference, AIAA-2017-4221, June 2017.

${ }^{15}$ Bisplinghoff, R. L., Ashley, H., and Halfman, R. L., Aeroelasticity, Addison-Wesley Publishing Company, Cambridge, MA, 1955.

${ }^{16}$ U.S. Department of Defense, "Flying Qualities of Piloted Airplanes," U.S. Military Specification MIL-F-8785C, Washington, D. C., 1980.

${ }^{17}$ Ly, U.-L. and Chan, Y. K., "Time-Domain Computation of Aircraft Gust Covariance Matrices, " AIAA Atmospheric Flight Mechanics Conference, AIAA-1980-1615, 1980.

${ }^{18}$ Jones, R. T., "Operational Treatment of the Nonuniform-Lift Theory in Airplane Dynamics," NACA TN 667, 1938.
} 